\title{
Fractional-Order Total Variation Combined with Sparsifying Transforms for Compressive Sensing Sparse Image Reconstruction
}

\author{
Gao Chen, Jiashu Zhang*, Defang Li
}

Sichuan Province Key Lab of Signal and Information Processing, Southwest Jiaotong University,

Chengdu 610031, People's Republic of China

\begin{abstract}
The total variation (TV) model has been considered to be one of the most successful and representative model for compressive sensing (CS) sparse image reconstruction due to its advantage of preserving image edges. However, TV regularized term often favors piecewise constant solution and therefore it fails to preserve the details and textures. To overcome this defect and reconstruct the fine details, this paper proposes a two-dimensional CS sparse image reconstruction model by introducing the fractional-order TV (FrTV) regularization constraint into CS optimization problem. Furthermore, in order to achieve sparser representation flexibly, a combination of discrete wavelet transform and curvelet transform $\ell_{1}$-norm regularization is also incorporated into the cost function and a method for estimating the regularization parameter that trades off the two terms in the cost function is proposed. By using a smooth approximation of the $\ell_{1}$-norm, a gradient projection algorithm is derived to solve the combined FrTV and sparsifying transforms constrained minimization problem effectively. Compared with several state-of-the-art reconstruction algorithms, the proposed algorithm is more efficient and robust, not only yielding higher peak-signal-to-noise ratio, but also reconstructing the fine details and textures more efficiently.
\end{abstract}

Keywords: two-dimensional compressive sensing; reconstruction; fractional-order total variation; gradient projection

\footnotetext{
* Corresponding author.

E-mail addresses: gchen984@gmail.com (G.Chen), jszhang@home.swjtu.edu.cn

(J.Zhang),ldf125@home.swjtu.edu.cn(D. Li).
} 


\section{Introduction}

Compressive sensing (CS) $[1,2]$ is a novel technique for signal acquiring, processing and compression. It states that as long as the signal is sparse or compressible, then we can roughly recover the original signal through only a few measurements, namely, we can achieve data sampling and compression at the same time. Due to its advantages of sampling below Nyquist rate and little loss in reconstruction quality, research on CS based image/video processing has become a hot topic in recent years[3,4,5].

Image sparse representation is a key factor that affects the quality of the reconstructed image. Total variation [6] (TV) regularization is one of the most successful sparse representation method for CS image reconstruction. It was first proposed for image denoising and since then had been successfully used for CS reconstruction[7,8,9,10]. However, the use of TV can not recover the fine details and textures, and often suffers from undesirable staircase artifact. Wavelet transform [11] is another good sparse representation for piecewise constant images. It is good at sparsely representing pointlike features but fails in sparsely representing the curve-like features[12]. Other geometric image transforms, such as curvelet [13] or contourlet[14], can sparsely represent curve-like features. But they are not good at representing point-like image features. In fact, each specific transform is often limited in that it may be optimal for one kind of features, but may be poor for other kinds of features. To overcome this shortage, a framework that combined these sparsifying transforms was proposed in [12] and [15] for CS magnetic resonance imaging (MRI). Nevertheless, the disadvantage of this method is that two (or more) regularization parameters need to be tuned. In our previous work[10], a new model for natural images sparse representation was proposed, in which the dual-tree discrete wavelet transform (DDWT) $\ell_{0}$-norm and the approximate TV norm were applied simultaneously. However, this method lacked 
rigorous theoretical analysis on the choice of the regularization parameter in the cost function.

Recently, a class of non-local fractional operator techniques has received considerable interests and is widely used in image denoising. In [16], a class of second-order and fourth-order anisotropic diffusion equations was proposed for noise removal, where a discrete Fourier transform was employed to evaluate the fractional differential values. In [17], a new space of function of fractional-order bounded variation was defined, based on this definition, a class of fractional-order multi-scale variational models and an alternating projection algorithm for image denoising was proposed. Based on these works, it can be confirmed that the fractional differential regularization model performs favorably for image denosing in terms of less blocky effect and detail preservation[18].

Inspired by the fractional operator techniques, in this paper a two-dimensional compressive sensing (2DCS) sparse image reconstruction model based on fractionalorder TV (FrTV) is proposed to reconstruct the fine details and textures of the images. The overall cost function for the proposed model consists of two terms, i.e., FrTV regularization and a combination of discrete wavelet transform and curvelet transform regularization. The role of the fractional order TV regularization term is to better handle the texture details of image, while the sparsifying transform regularization term is capable of preserving the point and curve features of images. A method for estimating the regularization parameter that trades off the two terms in the cost function is also proposed. Furthermore, by using a smooth approximation of the $\ell_{1}$-norm, a gradient projection algorithm FrTVGP (fractional-order TV gradient projection) is derived to solve this combined FrTV and sparsifying transforms constrained minimization problem effectively. The main advantages of our proposed algorithm include: (1) Unlike the 
existing TV-based models for CS image reconstruction, the proposed model does not suffer from block artifacts, staircase edges and false edge near the edges. (2) The proposed model can preserve edges sharply and smoothen regions better by taking advantage of FrTV and sparsifying transforms. (3) Compared with several state-of-theart CS based image reconstruction algorithms, the reconstructed images obtained by the proposed approach not only have higher peak-signal-to-noise (PSNR) values, but also have better visual effects. Numerous experiments are performed on natural images and demonstrate these advantages.

The rest of this paper is organized as follows. Section II introduces related concepts and works in the CS theory. In Section III, our FrTV-based two dimensional compressive sensing sparse image reconstruction algorithm is stated, discussing its sparseness modeling, reconstruction formulation. Experimental results are presented and discussed in Section IV. Finally some conclusions are given in Section V.

\section{Overview of $1 \mathrm{D}$ compressive sensing and $2 \mathrm{D}$ compressive sensing}

\section{$2.11 D$ compressive sensing}

In the standard CS framework, consider a discrete-time signal $\mathbf{x} \in R^{N}$ which has a sparse representation in some basis $\boldsymbol{\Psi} \in R^{N \times N}$ :

$$
\mathbf{x}=\sum_{i=1}^{N} \psi_{i} s_{i}=\boldsymbol{\Psi} \mathbf{s}, \quad K=\|\mathbf{s}\|_{0}, \quad K<<N
$$

Take $M$ linear measurements of $\mathbf{x}$ by projecting it onto some Gaussian measurement basis A of size $M \times N$, for some $K \log \left(\frac{N}{K}\right)<M<N$, i.e.:

$$
\mathbf{y}=\mathbf{A x}=\mathbf{A} \Psi \mathbf{s}=\mathbf{A}^{\mathrm{Cs}} \mathbf{s}
$$

Consequently, the information of $N$-dimensional signal is reduced to the $M$-dimensional measurement vector. 
Since the number of measurements $M<N$, the problem of reconstructing $\mathbf{x}$ from $\mathbf{y}$ is ill conditioned. However, the CS theory points out that if the measurement matrix A and the representation basis $\boldsymbol{\Psi}$ are sufficiently incoherent, we can reconstruct the original signal from the measurements exactly by solving an optimization problem:

$$
\begin{aligned}
& \min _{S}\|\mathbf{s}\|_{0} \\
& \text { s.t. } \quad \mathbf{A} \Psi \mathbf{s}=\mathbf{y}
\end{aligned}
$$

Unfortunately, $\ell_{0}$-norm minimization is not convex, and the computational complexity of optimizing it is non-polynomial (NP) hard. To overcome this difficulty, it was demonstrated that under certain condition, $\ell_{0}$-norm can be converted to the convex $\ell_{1}$ norm:

$$
\begin{aligned}
& \min _{S}\|\mathbf{s}\|_{1} \\
& \text { s.t. } \quad \mathbf{A} \Psi \mathbf{s}=\mathbf{y}
\end{aligned}
$$

where $\|\mathbf{s}\|_{1}$ is the $\ell_{1}$-norm of $\mathbf{s}$ defined as $\|\mathbf{s}\|_{1}=\sum_{i=1}^{N}\left|s_{i}\right|$. Many algorithms have been proposed to solve problem (3) and (4), typical algorithms include such as basis pursuit (BP)[19], matching pursuit (MP)[20], orthogonal matching pursuit (OMP)[21], and so on.

\section{$2.22 D$ compressive sensing}

When CS theory is applied to images, in order to collect a set of linear measurements of an image, columns of the image are first stacked into a large column vector, this vector space model for CS, needs prohibitive computational and memory requirements for collecting linear measurements and reconstruction algorithms after projection. Therefore, direct application of CS to 2D images involves prohibitive computational and memory requirements. 
In order to overcome this deficiency, some researchers extended CS to 2DCS[22,23]. Consider an image $\mathbf{X} \in R^{N \times N}$, directly leverage the matrix structure of $\mathbf{X}$ and take linear measurements of it by projecting it onto some Gaussian measurement basis $\mathbf{A}$ and $\mathbf{B}$ of size $M \times N(M<N)$ :

$$
\mathbf{Y}=\mathbf{A X B ^ { T }}
$$

where $\mathbf{A}, \mathbf{B}$ are $M \times N$ Gaussian random matrices, $\mathbf{Y} \in R^{M \times M}$ is the $2 \mathrm{D}$ measurements. This $2 \mathrm{D}$ measurement, in contrast to $1 \mathrm{D}$ measurement, requires only $2 M N$ memory units to store the measurement matrices.

\section{2D compressive sensing sparse image reconstruction based on fractional-order}

\section{total variation}

\subsection{Fractional-order total variation theory}

TV regularization is one of the most successful sparse representation method for CS image reconstruction. However, TV is based on the assumption that the signal is piecewise smooth. This assumption makes the TV regularization model suffer from oversmoothing, which means that TV cannot perfectly preserve details like texture and details. To alleviate this side effect of TV, many methods with high-order regularization items were proposed. Nevertheless, a high-order term will cause speckle-like noise[24]. To achieve a good trade off between them, we introduce a fractional-order regularization item into the $\mathrm{CS}$ image reconstruction algorithm.

A fractional-order gradient can be viewed as a generalization of the integer-order gradient composed of the fractional-order derivative of a different direction. Without loss of generality, let $\mathbf{X}_{i, j}$ denote the pixel in the $i_{\text {th }}$ row and $j_{\text {th }}$ column of a $N \times N$ image $\mathbf{X}$, the fractional-order discrete gradient transform $\nabla^{\alpha}: C^{N \times N} \rightarrow C^{N \times N \times 2}$ is defined in terms of the fractional-order derivatives in the directions of the columns and 
rows:

$$
\left[\nabla^{\alpha} \mathbf{X}\right]_{i, j} \stackrel{\text { def }}{=}\left(\left(\mathbf{X}_{h}^{\alpha}\right)_{i, j},\left(\mathbf{X}_{v}^{\alpha}\right)_{i, j}\right)
$$

where

$$
\left\{\begin{array}{l}
\left(\mathbf{X}_{h}^{\alpha}\right)_{i, j}=\sum_{k=0}^{K-1} W_{k}^{(\boldsymbol{\alpha})} \mathbf{X}(i-k, j) \\
\left(\mathbf{X}_{v}^{\alpha}\right)_{i, j}=\sum_{k=0}^{K-1} W_{k}^{(\boldsymbol{\alpha})} \mathbf{X}(i, j-k)
\end{array}\right.
$$

$W_{k}^{(\boldsymbol{\alpha})}=(-1)^{k}\left(\begin{array}{l}\alpha \\ k\end{array}\right)$ represents the coefficients of the polynomial $(1-z)^{\alpha}, K \geq 3$ is the number of terms involved in the computation of the fractional-order derivative and is usually set as $K=N$. Based on this definition, the FrTV semi-norm is defined as:

$$
\|\mathbf{X}\|_{F r T V}=\sum_{i=1}^{N} \sum_{j=1}^{N} \sqrt{\left[\left(\mathbf{X}_{h}^{\alpha}\right)_{i, j}\right]^{2}+\left[\left(\mathbf{X}_{v}^{\alpha}\right)_{i, j}\right]^{2}}
$$

Using the matrix approximate method, (7) can be written as the following form:

$$
\left\{\begin{array}{l}
\mathbf{X}_{\mathrm{h}}^{\alpha}=\mathbf{D}_{\mathrm{h}}^{\alpha} \cdot \mathbf{X} \\
\mathbf{X}_{\mathrm{v}}^{\alpha}=\mathbf{X} \cdot \mathbf{D}_{\mathbf{v}}^{\alpha}
\end{array}\right.
$$

where

$$
\begin{aligned}
& \mathbf{D}_{\mathbf{h}}^{\boldsymbol{\alpha}}=\left[\begin{array}{cccc}
W_{0}^{\boldsymbol{\alpha}} & 0 & \cdots & 0 \\
W_{1}^{\boldsymbol{\alpha}} & W_{0}^{\boldsymbol{\alpha}} & \cdots & 0 \\
\vdots & \vdots & \ddots & \vdots \\
W_{K-1}^{\boldsymbol{\alpha}} & W_{K-2}^{\boldsymbol{\alpha}} & \cdots & W_{0}^{\boldsymbol{\alpha}}
\end{array}\right] \\
& \mathbf{D}_{\mathbf{v}}^{\boldsymbol{\alpha}}=\left[\begin{array}{cccc}
W_{0}^{\boldsymbol{\alpha}} & W_{1}^{\boldsymbol{\alpha}} & \cdots & W_{K-1}^{\boldsymbol{\alpha}} \\
0 & W_{0}^{\boldsymbol{\alpha}} & \cdots & W_{K-2}^{\boldsymbol{\alpha}} \\
\vdots & \vdots & \ddots & \vdots \\
0 & 0 & \cdots & W_{0}^{\boldsymbol{\alpha}}
\end{array}\right]
\end{aligned}
$$

Then the approximate FrTV can be rewritten as: 


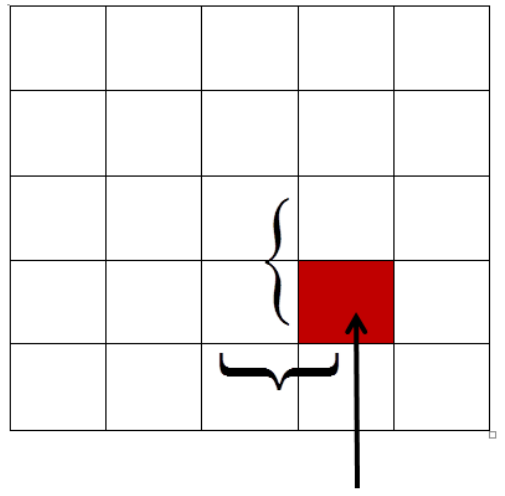

$\left(\nabla \mathbf{X}_{h}\right)_{4,4}=\mathbf{X}_{4,4}-\mathbf{X}_{3,4}$

$\left(\nabla \mathbf{X}_{v}\right)_{4,4}=\mathbf{X}_{4,4}-\mathbf{X}_{4,3}$

(a)

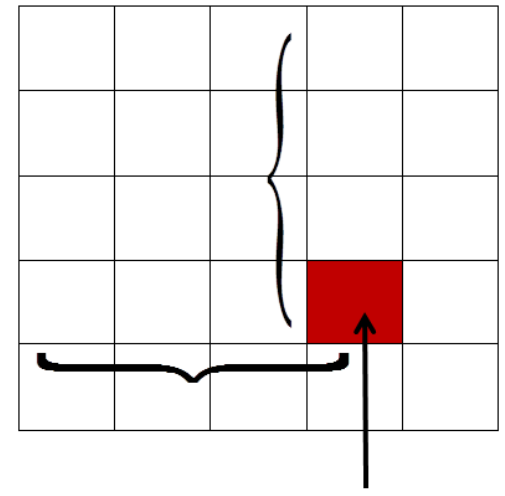

$\left(\nabla \mathbf{X}_{h}^{\alpha}\right)_{4,4}=W_{0}^{\alpha} \mathbf{X}_{4,4}+W_{1}^{\alpha} \mathbf{X}_{3,4}+W_{2}^{\alpha} \mathbf{X}_{2,4}+W_{3}^{\alpha} \mathbf{X}_{1,4}$

$\left(\nabla \mathbf{X}_{v}^{\alpha}\right)_{4,4}=W_{0}^{\alpha} \mathbf{X}_{4,4}+W_{1}^{\alpha} \mathbf{X}_{4,3}+W_{2}^{\alpha} \mathbf{X}_{4,2}+W_{3}^{\alpha} \mathbf{X}_{4,1}$

(b)

Fig.1 (a)Integer-order derivative (b)Fractional-order derivative

$$
\operatorname{FrTV}(\mathbf{X})=\sum_{i=1}^{N} \sum_{j=1}^{N} \sqrt{\left(\mathbf{D}_{\mathbf{h}}^{\alpha} \cdot \mathbf{X}\right)_{i, j}^{2}+\left(\mathbf{X} \cdot \mathbf{D}_{\mathbf{v}}^{\alpha}\right)_{i, j}^{2}}
$$

There are two main reasons why the FrTV performs better than traditional TV for CS reconstruction. First, as shown in (7) and Fig.1, unlike integer-order derivative, fractional-order derivative derivative at pixel $\mathbf{X}_{i, j}$ is not determined by a small neighborhood of $\mathbf{X}_{i, j}$, in other words, fractional-order derivative is not a local property of an image. This property may enable fractional-order derivative to deal well with texture information since texture is not local in nature. It can enhance texture details in those areas where gray-level values do not change evidently. Second, both integer-order and fractional-order differentiations can enhance high-frequency components, but the enhancement of integer-order differentiation is stronger than that of fractional-order differentiation, as shown in [25]. Consequently, fractional-order differentiation introduces relatively low contrast and avoids very large oscillations near edges. 
Fig. 2 shows the fractional-order derivative picture in horizontal direction and vertical direction of image Lena and their histograms. It is obvious to see that the distribution is very sharp and most pixels values are near zero.
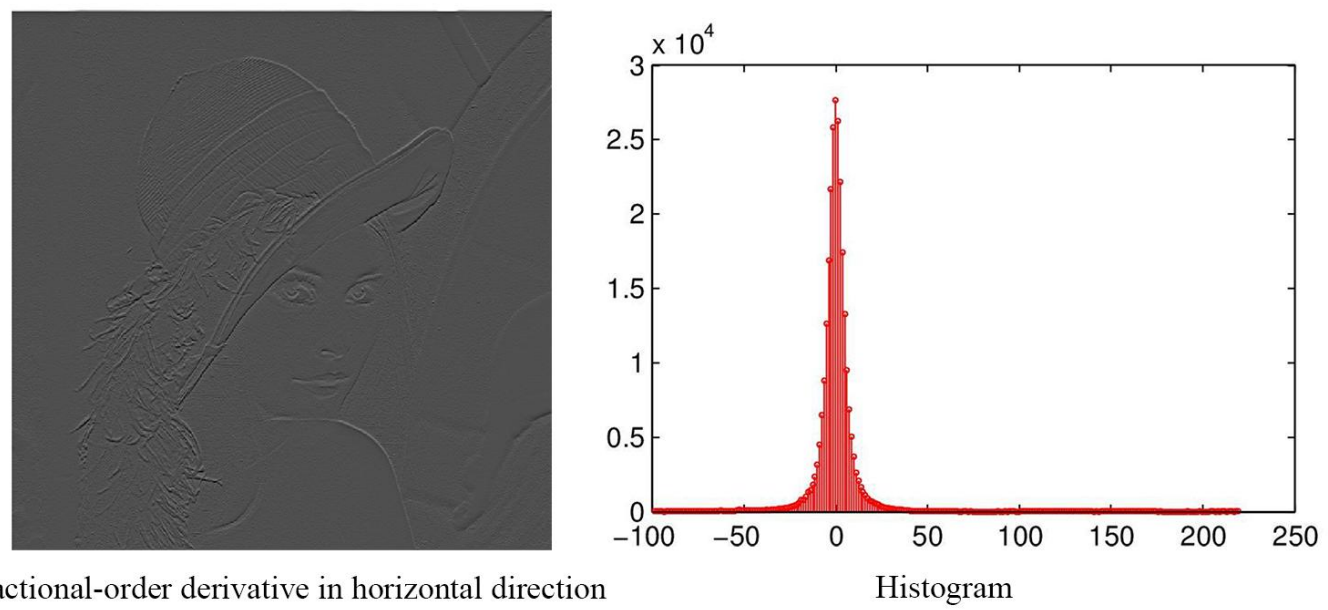

Fractional-order derivative in horizontal direction

Histogram

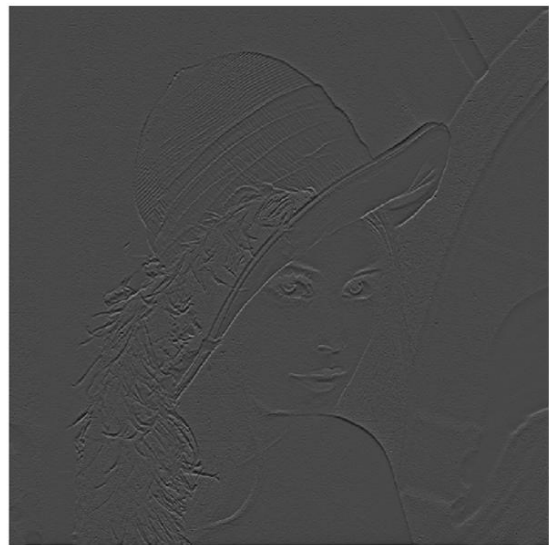

Fractional-order derivative in vectical direction

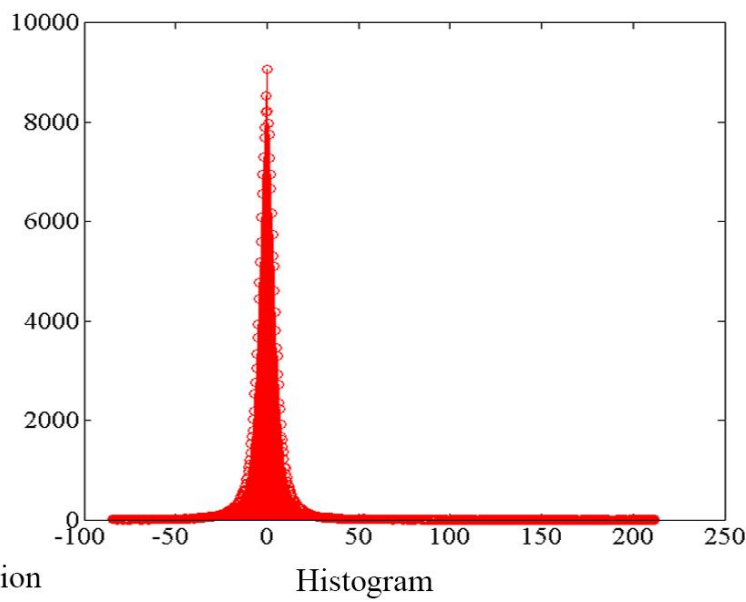

Fig.2 Fractional-order derivative pictures of image Lena and their histograms.

\subsection{Sparseness model for image reconstruction}

As mentioned in section I, natural images are of morphological diversities and are often rich in textures and fine details. DWT is good at sparsely representing point-like features but fails in sparsely representing the curve-like features. Curvelet or contourlet can sparsely represent curve-like features. But they are not good at representing pointlike image features. In fact, each specific transform is often limited in that it may be 
optimal for one kind of features, but may be poor for other kinds of features. To overcome this shortage, we combine DWT and curvelet transform to construct an overcomplete dictionary and use this dictionary to sparsely represent the natural images.

The combination of discrete wavelet transform and curvelet transform of an image $\mathbf{X}$ can be formulated as follows:

$$
\mathbf{S}=\mathbf{W v e c}(\mathbf{X})
$$

where $\mathbf{W}=\left[\mathbf{W}_{1}^{\mathbf{T}}, \mathbf{W}_{2}^{\mathbf{T}}\right]^{\mathbf{T}}, \mathbf{W}_{\mathbf{1}}$ is the discrete wavelet transform matrix, $\mathbf{W}_{2}$ is the curvelet transform matrix. $\mathbf{W}_{1}$ and $\mathbf{W}_{2}$ can be constructed by the Spot Matlab toolbox [26]. Fig.3 shows the histogram of the transform coefficient $\mathbf{S}$ of image Lena. It is obvious to see that the most values of $\mathbf{S}$ are near zero.

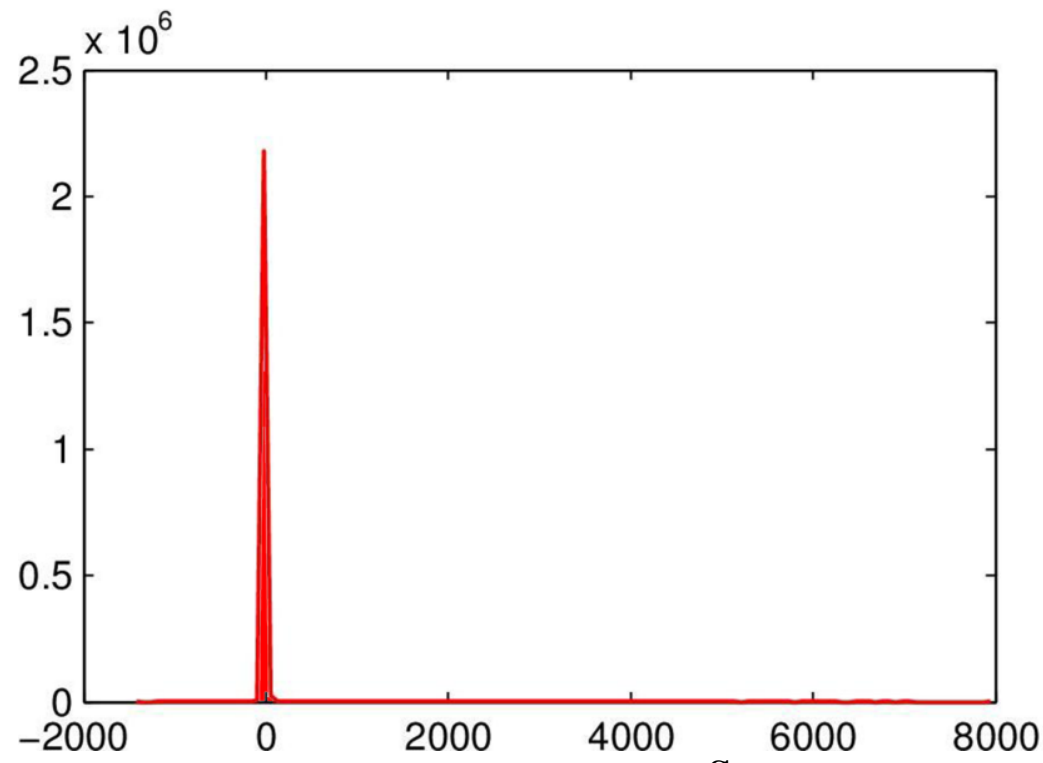

Fig.3 Histogram of the transform coefficient $\mathbf{S}$ of image Lena.

Combining (12) and (13), our 2DCS sparse image reconstruction based on FrTV model can be formulated as:

$$
\begin{aligned}
& \min _{\mathbf{X}}\|\mathbf{W} \operatorname{vec}(\mathbf{X})\|_{1}+\beta \sum_{i=1}^{N} \sum_{j=1}^{N} \sqrt{\left(\mathbf{D}_{\mathbf{h}}^{\alpha} \cdot \mathbf{X}\right)_{i, j}^{2}+\left(\mathbf{X} . \mathbf{D}_{\mathbf{v}}^{\alpha}\right)_{i, j}^{2}} \\
& \text { s.t. } \quad \mathbf{Y}=\mathbf{A} \mathbf{X B}^{T}
\end{aligned}
$$


where $\beta$ is a positive regularization parameter to trade off the sparsity in the combined sparsifying transform domain and in the FrTV domain. We will discuss how to set this parameter in section 3.4

\subsection{Reconstruction algorithm}

The reconstruction model in (14) consists of a smooth FrTV norm and a non-smooth $\ell_{1}$-norm. As a result, it does not have a defined gradient at all points that have at least one zero component. In this section, by using a smooth approximation of the $\ell_{1}$-norm, a gradient projection algorithm FrTVGP is developed to solve this underdetermined minimization problem effectively.

There are numerous smooth approximations of $\ell_{1}$-norm. A popular approximation [27] is:

$$
\|\mathbf{x}\|_{1} \approx \frac{1}{T} \sum_{i=1}^{N} \ln \left[\cosh \left(T \mathbf{x}_{i}\right)\right]
$$

where $T \gg>1$ is a tuning parameter, $N$ is the length of the vector $\mathbf{x}$. Using this $\ell_{1}$-norm approximate method, problem (14) can be written as:

$$
\begin{aligned}
& \min \quad F(\mathbf{X})=f_{1}(\mathbf{X})+\beta f_{2}(\mathbf{X}) \\
& =\frac{1}{T} \sum_{i=1}^{N_{1}} \ln \left\{\cosh \left[T(\mathbf{W} \operatorname{vec}(\mathbf{X}))_{i}\right]\right\}+\beta \sum_{i=1}^{N} \sum_{j=1}^{N} \sqrt{\left(\mathbf{D}_{\mathbf{h}}^{\alpha} \cdot \mathbf{X}\right)_{i, j}^{2}+\left(\mathbf{X} \cdot \mathbf{D}_{\mathbf{v}}^{\alpha}\right)_{i, j}^{2}} \\
& \text { s.t. } \mathbf{Y}=\mathbf{A X B}^{T}
\end{aligned}
$$

where $N_{1}$ is the length of the vector $\mathbf{W v e c}(\mathbf{X})$.

After this approximation, problem (16) can be solved by general gradient descent projection method, the implementation details of minimizing $F(\mathbf{X})$ with general gradient descent projection are given below.

\subsubsection{Initialization}


In FrTVGP algorithm, the initial value is the minimum $\ell_{2}$-norm solution of $\mathbf{Y}=\mathbf{A X B} \mathbf{B}^{\mathbf{T}}$. The minimum $\ell_{2}$-norm solution of $\mathbf{Y}=\mathbf{A} \mathbf{X B} \mathbf{B}^{\mathbf{T}}$ is:

$$
\mathbf{X}^{0}=\mathbf{A}^{+} \mathbf{Y}\left(\mathbf{B}^{+}\right)^{T}
$$

where $\mathbf{A}^{+}=\mathbf{A}^{T}\left(\mathbf{A} \mathbf{A}^{T}\right)^{-1}$ and $\mathbf{B}^{+}=\mathbf{B}^{T}\left(\mathbf{B} \mathbf{B}^{T}\right)^{-1}$ are the pseudoinverses of $\mathbf{A}$ and $\mathbf{B}$

\subsubsection{Gradient descent}

The derivative of $F(\mathbf{X})$ with respect to the matrix $\mathbf{X}$ is given by:

$$
\begin{aligned}
& \frac{\partial F(\mathbf{X})}{\partial \mathbf{X}}=\frac{\partial f_{1}(\mathbf{X})}{\partial \mathbf{X}}+\beta \frac{\partial f_{2}(\mathbf{X})}{\partial \mathbf{X}} \\
& \frac{\partial f_{1}(\mathbf{X})}{\partial \mathbf{X}}=\operatorname{mat}_{N, N}\left\{\mathbf{W}^{\mathbf{T}} \tanh [T \mathbf{W} \operatorname{vec}(\mathbf{X})]\right\} \\
& \frac{\partial f_{2}(\mathbf{X})}{\partial \mathbf{X}}=\left(\mathbf{D}_{\mathbf{h}}^{\alpha}\right)^{T} \mathbf{E}+\mathbf{F}\left(\mathbf{D}_{\mathbf{v}}^{\alpha}\right)^{T}
\end{aligned}
$$

where $\operatorname{mat}_{N, N}(\bullet)$ reverses the operation of vectorization, i.e., $\operatorname{mat}_{N, N}(\operatorname{vec}(\mathbf{X}))=\mathbf{X}$

$$
\begin{aligned}
& \mathbf{E}(i, j)=\mathbf{C}_{\mathbf{1}}(i, j) \mathbf{H}(i, j) \\
& \mathbf{F}(i, j)=\mathbf{C}_{2}(i, j) \mathbf{H}(i, j) \\
& \mathbf{C}_{\mathbf{1}}=\mathbf{D}_{\mathbf{h}}^{\alpha} \cdot \mathbf{X} \\
& \mathbf{C}_{\mathbf{2}}=\mathbf{X} \cdot \mathbf{D}_{\mathbf{v}}^{\alpha} \\
& \mathbf{H}(i, j)=\frac{1}{\sqrt{\left[\mathbf{C}_{\mathbf{1}}(i, j)\right]^{2}+\left[\mathbf{C}_{\mathbf{2}}(i, j)\right]^{2}}}
\end{aligned}
$$

Then the gradient descent recursion of $\mathbf{X}$ for $F(\mathbf{X})$ can be written as:

$$
\hat{\mathbf{X}}_{n}=\mathbf{X}_{n}-\lambda \frac{\partial F\left(\mathbf{X}_{n}\right)}{\partial \mathbf{X}_{n}}
$$

where $\lambda$ is the step-size.

\subsubsection{Projection}


After the gradient descent, the iterative solution departs from the solution space, so the updated matrix is replaced by the projection of the matrix on the solution space. Suppose that $\widehat{\mathbf{X}}_{n+1}$ is the result gained after the gradient descent, $\mathbf{X}_{n+1}$ is its projection matrix in the solution space, $\tilde{\mathbf{X}}$ is a variable, the projection is realized by solving an optimization problem: finding a $\tilde{\mathbf{X}}$ that satisfies $\mathbf{Y}=\mathbf{A} \tilde{\mathbf{X}} \mathbf{B}^{T}$ and $\left\|\tilde{\mathbf{X}}-\hat{\mathbf{X}}_{n+1}\right\|_{F}^{2}$ reaches the minimum, it can be formulated as follows:

$$
\begin{aligned}
& \mathbf{X}_{n+1}=\underset{\tilde{\mathbf{X}}}{\arg \min }\left\|\tilde{\mathbf{X}}-\hat{\mathbf{X}}_{n+1}\right\|_{F}^{2} \\
& \text { s.t. } \quad \mathbf{Y}=\mathbf{A} \tilde{\mathbf{X}} \mathbf{B}^{T}
\end{aligned}
$$

Laplacian Method can be used to solve(27):

$$
\mathbf{X}_{n+1}=\widehat{\mathbf{X}}_{n+1}-\mathbf{A}^{+}\left(\mathbf{A} \widehat{\mathbf{X}}_{n+1} \mathbf{B}^{\mathbf{T}}-\mathbf{Y}\right)\left(\mathbf{B}^{+}\right)^{T}
$$

\subsection{The choice of regularization parameter}

There is a positive regularization parameter $\beta$ in our proposed reconstruction model(14), which controls the balance between the sparsity in the combined sparsifying transform domain and in the FrTV domain. Different from the other works that set this parameter by simply searching, a new method for estimating this regularization parameter is proposed in this section. The basic idea of this method is to utilize the information available at each iteration step in the reconstruction process.

We find that the regularization parameter $\beta$ has the following properties:

1) $\beta$ is proportional to $\|\mathbf{W} v e c(\mathbf{X})\|_{1}$, a large $\|\mathbf{W} v e c(\mathbf{X})\|_{1}$ means that the reconstructed image is not sparse, thus a large regularization parameter should be selected to make sure that the reconstructed image is sparse in the wavelet domain. 
Algorithm 1: Fractional-order TV gradient projection algorithm

Input: $\mathbf{Y}, \mathbf{A}, \mathbf{B}, \varepsilon, \alpha, \lambda, \xi, \mathrm{T}, N_{0}$

- Initialization

- Let $n=0, \mathbf{X}^{0}=\mathbf{A}^{+} \mathbf{Y}\left(\mathbf{B}^{+}\right)^{T}$

- While $\left\|\mathbf{X}_{n}-\mathbf{X}_{n-1}\right\|_{F} \geq \xi$ or $n<N_{0}$

- $\tilde{\mathbf{X}}=\mathbf{X}_{n}-\lambda\left[\frac{\partial f_{1}(\mathbf{X})}{\partial \mathbf{X}}+\beta_{n} \frac{\partial f_{2}(\mathbf{X})}{\partial \mathbf{X}}\right]$

- $\mathbf{X}_{n+1}=\tilde{\mathbf{X}}-\mathbf{A}^{+}\left(\mathbf{A} \tilde{\mathbf{X}} \mathbf{B}^{\mathbf{T}}-\mathbf{Y}\right)\left(\mathbf{B}^{+}\right)^{T}$

- $\beta_{n+1}=\ln \left(\varepsilon \frac{\left\|\mathbf{W} \operatorname{vec}\left(\mathbf{X}_{n}\right)\right\|_{1}}{\operatorname{FrTV}\left(\mathbf{X}_{n}\right)+\delta}+1\right)$

$n=n+1$

- End while

- Output $\mathbf{X}_{n}$

2) $\beta$ is inversely proportional to $\operatorname{FrTV}(\mathbf{X})$, a large $\operatorname{FrTV}(\mathbf{X})$ means that the reconstructed image is not smooth and has little edge and detail information, thus a small regularization parameter should be selected to make sure that the reconstructed image is smooth.

3) $\beta$ is larger than zero.

To satisfy the above conditions, a regularization function is designed as follows: 


$$
\beta_{n+1}=\ln \left(\varepsilon \frac{\left\|\mathbf{W} \operatorname{vec}\left(\mathbf{X}_{n}\right)\right\|_{1}}{\operatorname{FrTV}\left(\mathbf{X}_{n}\right)+\delta}+1\right)
$$

where $\delta$ is a small positive number used to avoid a zero denominator, assigned $10^{-7}$ in practice, $\varepsilon$ is the modified factor of $\beta$.

The procedures of our reconstruction algorithm FrTVGP can be summarized in Table 1

\subsection{Computational complexity analysis}

In this subsection, we give the computational complexity analysis of the proposed FrTVGP algorithm.

The computational complexity of the initialization stage of the FrTVGP algorithm is implemented by $\mathbf{X}_{0}=\mathbf{A}^{\dagger} \mathbf{Y}\left(\mathbf{B}^{\dagger}\right)^{T}$, the complexity of it is dominated by $(N \times M) \times(M \times M)$ matrix-matrix multiplication and $(N \times M) \times(M \times N)$ matrix-matrix multiplication. The first matrix-matrix multiplication needs $M^{2} N$ multiplications and the second matrix-matrix multiplication needs $M N^{2}$ multiplications, as $M<N$, the complexity of this step is $O\left(M N^{2}\right)$.

The computational complexity of gradient descent stage is implemented by (18), it needs $N^{3}$ matrix-matrix multiplications, so the complexity of this step is $O\left(N^{3}\right)$

The computational complexity of projection stage is implemented by (28), computing $\mathbf{A}^{+}\left(\mathbf{A} \widehat{\mathbf{X}}_{n+1} \mathbf{B}^{\mathrm{T}}-\mathbf{Y}\right)\left(\mathbf{B}^{+}\right)^{T}$ needs $M^{2} N+M N^{2}$ multiplications, so the complexity of this step is $O\left(M N^{2}\right)$

The computational complexity of updating the regularization parameter stage is dominated by computing the FrTV norm and the $\ell_{1}$ norm of a $N \times N$ matrix, so the complexity is $O\left(N^{2}\right)$ 


\section{Experimental results}

In this section, the effectiveness of the proposed algorithm is demonstrated via comprehensive experiments. The experiments are tested on several gray-level natural images with size $512 \times 512$, such as, Lena, Goldhill, Barbara and Peppers. These test images are shown in Fig.4. It is observed that there are a large mount of textures and fine details in images, especially in Barbara. In our experiments, the CS measurements are obtained by applying a Gaussian random projection matrix to the original image signal, where $M \times N$ matrices $\mathbf{A}$ and $\mathbf{B}$ in (14) are generated by Matlab command $\operatorname{randn}(M, N)$. Both peak signal to noise ratio (PSNR) and structural similarity indexes (SSIM) [28] are employed to evaluate the objective quality of the reconstructed images. PSNR is defined as:

$$
P S N R=20 \log _{10} R / R M S E
$$

with

$$
R M S E=\sqrt{\sum_{i=1}^{N} \sum_{j=1}^{N}\left(\mathbf{I}_{i j}-\widehat{\mathbf{I}}_{i j}\right)^{2}}
$$

where $\mathbf{I}_{i j}$ and $\widehat{\mathbf{I}}_{i j}$ denote the pixel values of the reconstructed image and the original image, respectively. $R>0$ is the maximum value of the image gray level range. SSIM is defined as:

$$
\operatorname{SSIM}(\mathbf{I}, \hat{\mathbf{I}})=\frac{\left(2 \mu_{\mathbf{x}} \mu_{\mathbf{Y}}+C_{1}\right)\left(2 \sigma_{\mathbf{X Y}}+C_{2}\right)}{\left(\mu_{\mathbf{x}}{ }^{2}+\mu_{\mathbf{Y}}{ }^{2}+C_{1}\right)\left(\sigma_{\mathbf{x}}^{2}+\sigma_{\mathbf{Y}}^{2}+C_{2}\right)}
$$

where $\mu_{\mathbf{x}}$ and $\mu_{\mathbf{Y}}$ are the mean value of image $\mathbf{I}$ and $\hat{\mathbf{I}}, \sigma_{\mathbf{x}}$ and $\sigma_{\mathbf{Y}}$ are the standard deviation of image $\mathbf{I}$ and $\mathbf{I}$ respectively, the constant $C_{1}$ and $C_{2}$ are included to avoid instability when $\mu_{\mathbf{x}}{ }^{2}+\mu_{\mathbf{Y}}{ }^{2}$ and $\sigma_{\mathbf{X}}^{2}+\sigma_{\mathbf{Y}}^{2}$ are very close to zero, and 


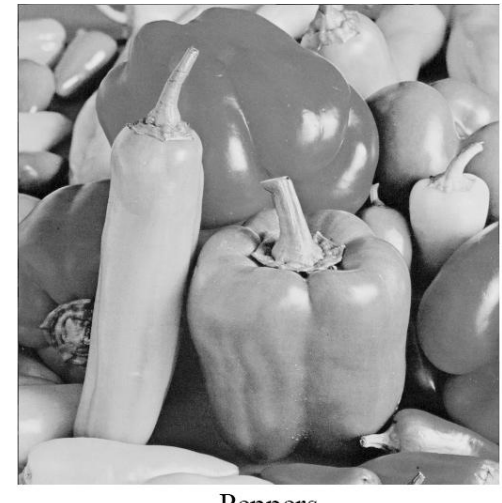

Peppers

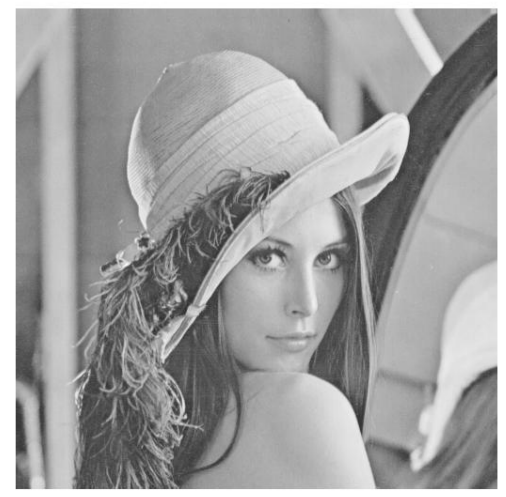

Lena

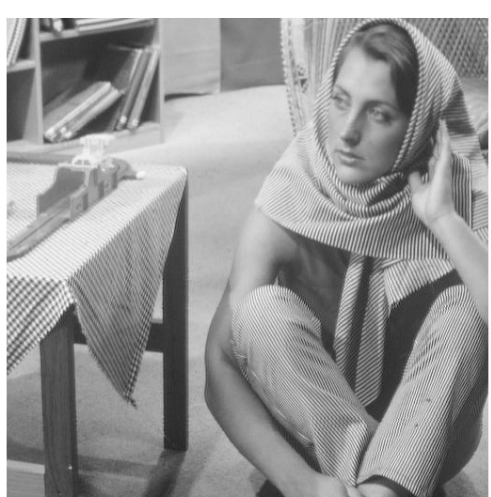

Barbara

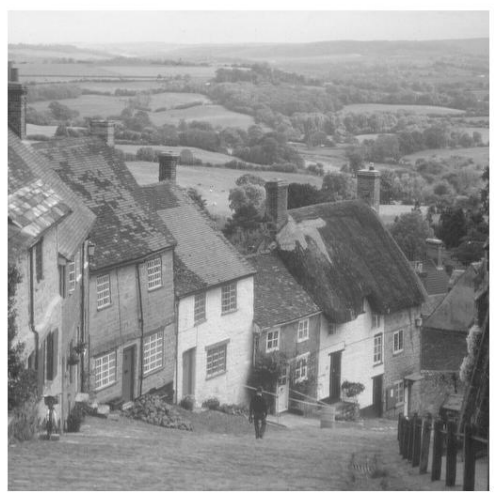

Goldhill

Fig.4 Four original test images used to simulate the algorithms

$$
\sigma_{\mathbf{X Y}}=\left(\frac{1}{N-1}\right)^{2} \sum_{i=1}^{N} \sum_{j=1}^{N}\left(\mathbf{I}_{i j}-\mu_{\mathbf{X}}\right)\left(\widehat{\mathbf{I}}_{i j}-\mu_{\mathbf{Y}}\right)
$$

\subsection{Impact of the parameters}

There are several parameters in our proposed algorithm, $\xi$ and $N_{0}$ are used to control the stop criterion, in our experiments, we set $\xi=10^{-5}, N_{0}=2000 . \varepsilon$ is the modified factor of the regularization parameter $\beta$, we empirically set $\varepsilon=0.4$ for all test images. It is necessary to stress that the choice for this parameter is general, and can be generalized to other natural images, which has been verified in our experiments. In our future research, we will explore some adaptive methods of selecting the optimal value of this parameter.

\subsubsection{The influence of parameter $T$}


$T$ is a tuning parameter of the smooth approximations of $\ell_{1}$ norm in (16), according to the conclusions of [27], it should satisfy $T>>1$. In this experiment, the effect of this parameter on the image reconstruction performance of the proposed algorithm is tested. The other parameters are set as: $\lambda=0.1, \alpha=1.4$ and the measurement rate is $50 \%$. Fig.5 displays the PSNR values with different $T$ for each test image. It can be seen from this figure that when $1<T<20$, the larger the parameter $T$ is, the higher recovery quality it will have. However, when $T>20$, it has little influence to the quality of the recovered images. That is because when $1<T<20$, the larger value of $T$, the better approximation to $\ell_{1}$ norm and when $T>>1$, the approximation tends to equality.

\subsubsection{The influence of fractional-order $\alpha$}

$\alpha$ is the fractional order of the FrTV, in this experiment, the effect of this parameter on the image reconstruction performance of the proposed algorithm is tested. We use Barbara as the test image and different fractional order $\alpha$ are chosen. The other

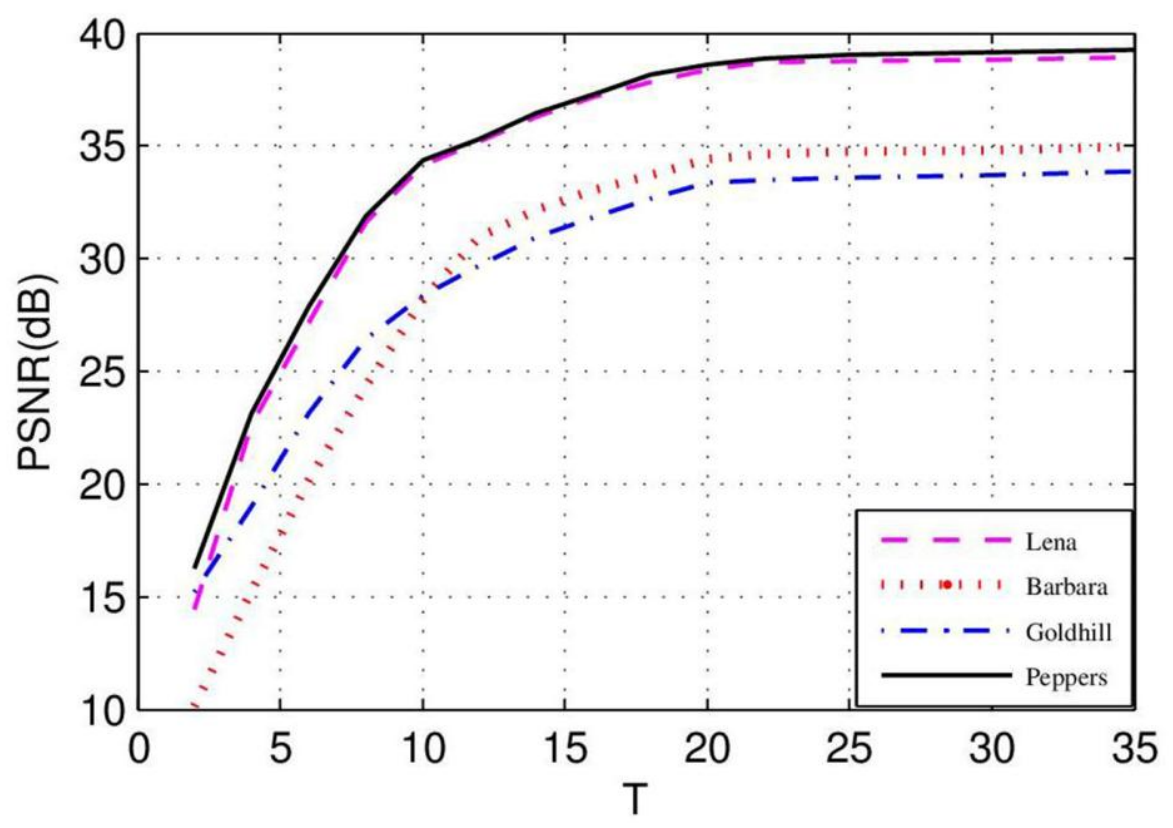

Fig.5 PSNR values with different $T$ for each test image 
parameters are set as: $\lambda=0.1, T=100$. Fig. 6 shows the reconstructed images and their local enlargements with different fractional order $\alpha$ under 50\% measurement rate. Fig.7 displays the PSNR values with the growth of the measurement rate for Barbara with different $\alpha$. From Fig.6 and Fig.7 we can see that when $\alpha<1$, the images reconstructed by the FrTV model lose more textures and details than TV model. When $\alpha=1$, FrTV is the traditional TV model. When $\alpha>1$, the larger the parameter $\alpha$ is, the better the textures are preserved. However, when $\alpha$ is close to 2, the frequency of textures will be enhanced excessively and become a kind of noise. To achieve a good tradeoff, in our experiments, we set $\alpha=1.4$.
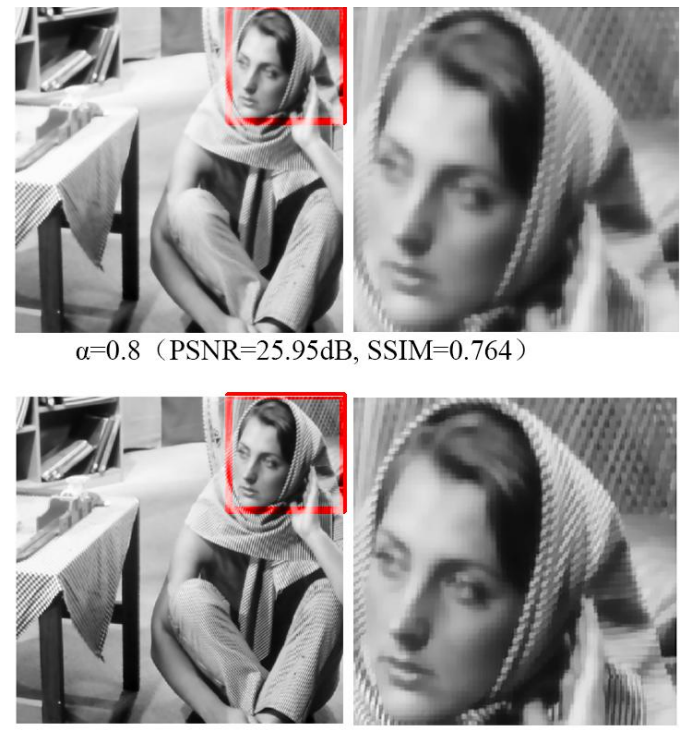

$\alpha=1.2 （ \operatorname{PSNR}=32.11 \mathrm{~dB}, \mathrm{SSIM}=0.911)$

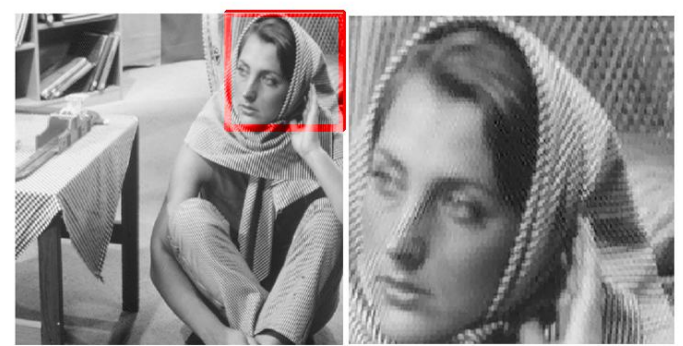

$\alpha=1.6(\operatorname{PSNR}=33.44 \mathrm{~dB}, \mathrm{SSIM}=0.926)$

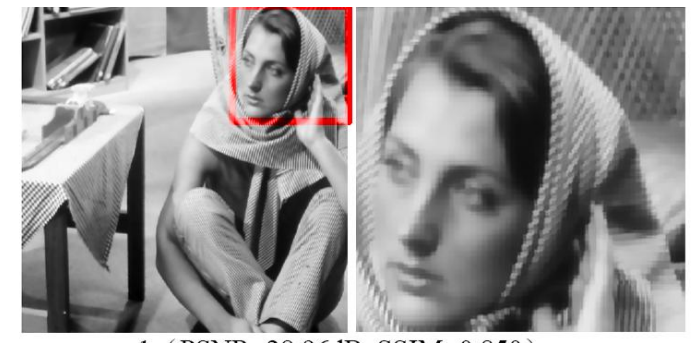

$\alpha=1 \quad(\operatorname{PSNR}=28.96 \mathrm{~dB}, \operatorname{SSIM}=0.850)$

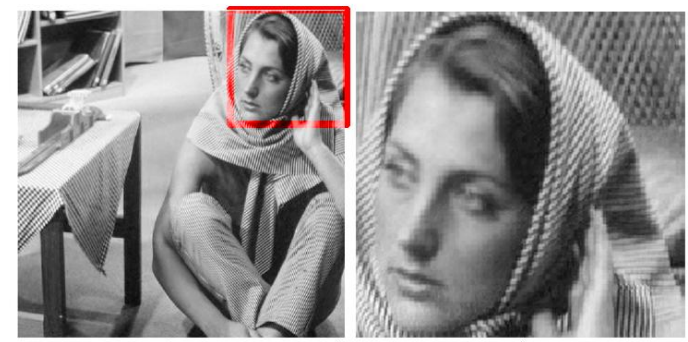

$\alpha=1.4(\operatorname{PSNR}=34.74 \mathrm{~dB}, \mathrm{SSIM}=0.935)$

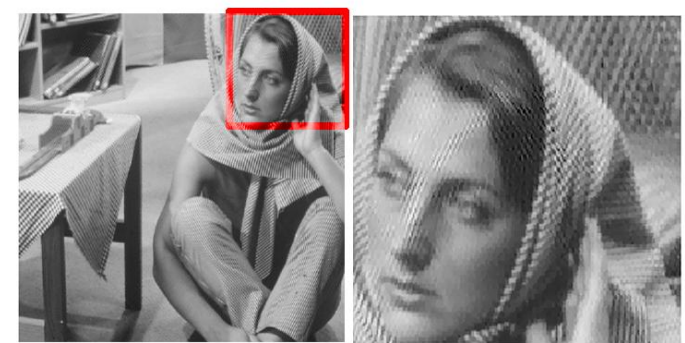

$\alpha=1.8($ PSNR $=30.39 \mathrm{~dB}, \mathrm{SSIM}=0.882)$

Fig 6 Reconstructed images (Barbara) with different fractional order $\alpha$, the measurement rate is $50 \%$

\subsubsection{The influence of the step-size $\lambda$}




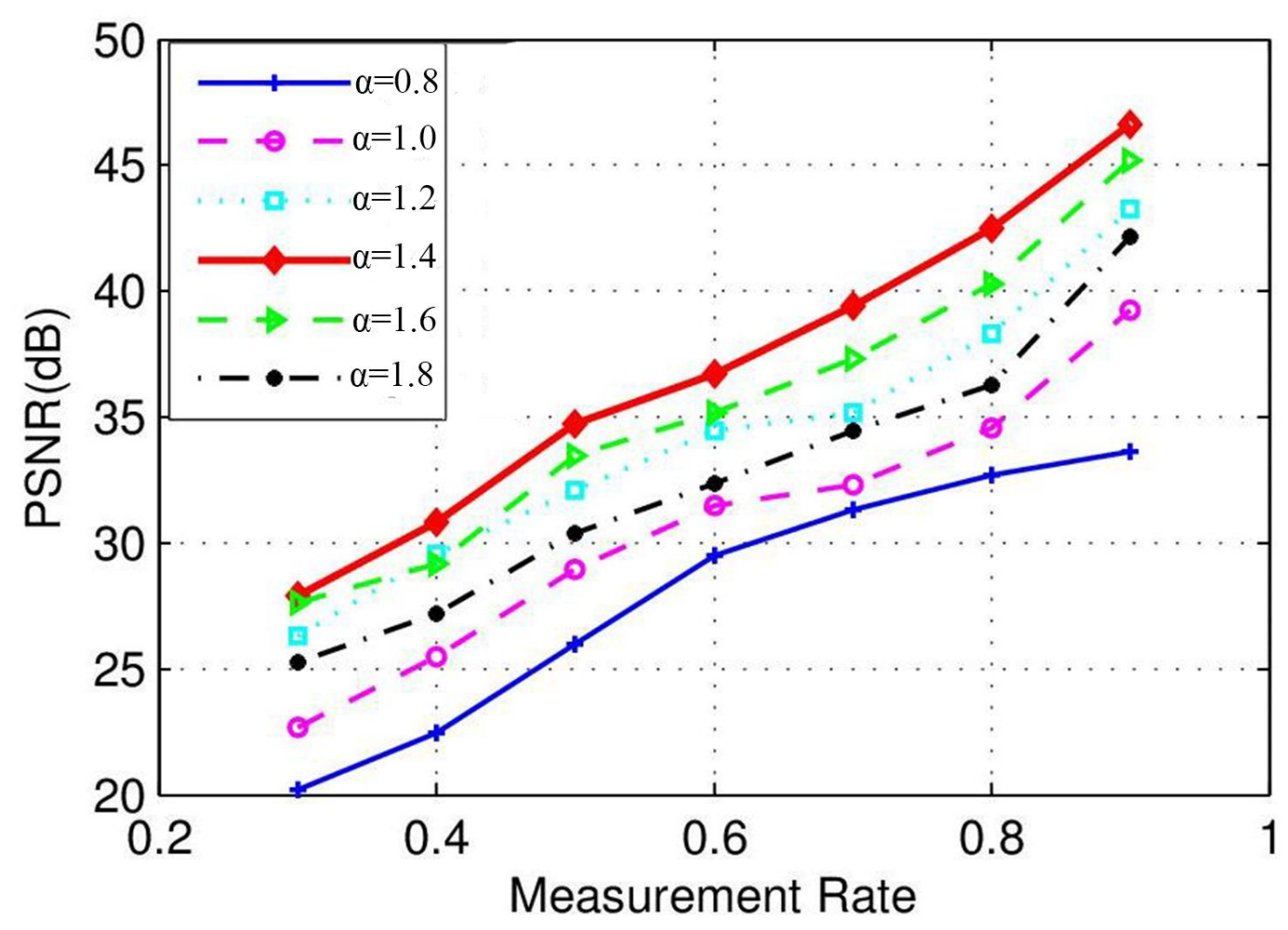

Fig.7 PSNR values with the growth of the measurement rate for Barbara with different $\alpha$.

In this experiment the effect of parameter $\lambda$ on the image reconstruction performance of the proposed algorithm is tested. We use Lena as the test image and different $\lambda$ are chosen. For each $\lambda$, we use the FrTVGP algorithm to reconstruct the image. The other parameters are set as: $T=100, \alpha=1.4$ and the measurement rate is $50 \%$ The convergence curves are shown in Fig.8. It can be seen from the figure that a large stepsize will result in a large derivation but a fast convergence. However, a small will induce a slower convergence but a smaller derivation. That is because a large step-size denotes that the sparsity is dominating, and it is expected that the reconstruction would have a quicker convergence. However, a too big value will break the balance between the sparisfy and the constraint, and the data fidelity will be affected. The reconstructed image may have a comparative sparsity, but the image is quite different from the original image. That is because the data fidelity in the reconstruction is sacrificed to 


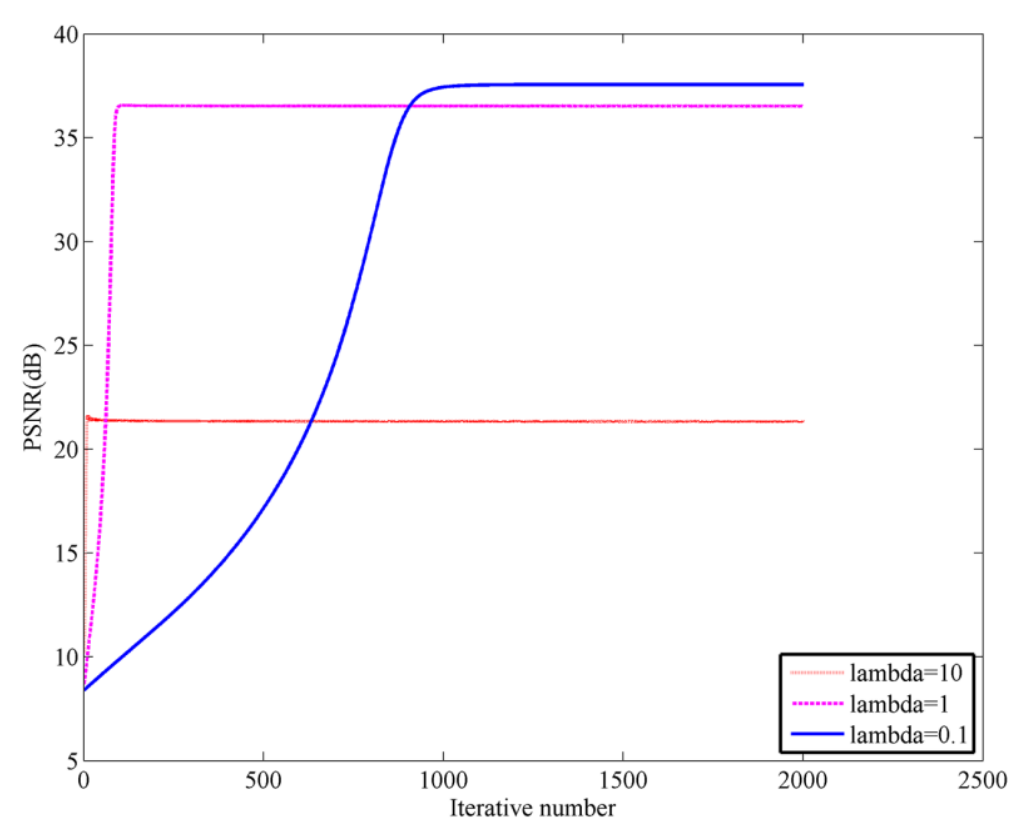

Fig.8 Convergence performances of FrTVGP under different step-size

image regularity. The quality of the reconstruction declines very much. Therefore, the parameter is determined by a tradeoff between convergence rate and reconstruction quality in particular applications.

\subsection{Comparison to other sparseness model}

Our proposed CS model includes a combination of sparsifying transforms $W$ and a FrTV model. In this section, the effect of sparsifying transforms on the image reconstruction performance and the superiority of the FrTV are tested.

\subsubsection{Compared with other sparsifying transforms}

In this experiment, the quality of the images reconstructed by the proposed algorithm using the FrTV combined with other sparsifying transforms is tested. The parameters are set as: $T=100, \alpha=1.4, \lambda=0.1$ and the measurement rate is $50 \%$. For each test image, the experiment is repeated ten times under different sampling matrix. Then, the average PSNR, SSIM, recovery time and their standard deviation are calculated and 
Table 2 The reconstruction performance using different sparsifying transforms combined with FrTV

\begin{tabular}{|c|c|c|c|c|}
\hline Image & Sparsifying model $\mathbf{W}$ & $\operatorname{PSNR}(\mathrm{dB})$ & SSIM & Recovery time(Sec) \\
\hline \multirow{7}{*}{ Barbara } & DCT & $25.72( \pm 0.26)$ & $0.786( \pm 0.023)$ & $52.05 \pm(1.24)$ \\
\hline & DWT & $28.49( \pm 0.34)$ & $0.843( \pm 0.012)$ & $40.78 \pm(1.31)$ \\
\hline & Curvelet & $30.44( \pm 0.42)$ & $0.884( \pm 0.020)$ & $100.3 \pm(1.27)$ \\
\hline & DWT+DCT & $32.07( \pm 0.78)$ & $0.899( \pm 0.019)$ & $103.2 \pm(1.56)$ \\
\hline & DCT +Curvelet & $32.36( \pm 0.33)$ & $0.921( \pm 0.014)$ & $225.9 \pm(1.21)$ \\
\hline & DWT +Curvelet & $35.37( \pm 0.47)$ & $0.939( \pm 0.011)$ & $207.3 \pm(1.14)$ \\
\hline & DWT+DCT+Curvelet & $36.08( \pm 0.39)$ & $0.944( \pm 0.010)$ & $365.4 \pm(1.28)$ \\
\hline \multirow{7}{*}{ Lena } & DCT & $30.25( \pm 0.26)$ & $0.833( \pm 0.017)$ & $51.57 \pm(1.36)$ \\
\hline & DWT & $32.96( \pm 0.18)$ & $0.849( \pm 0.019)$ & $40.23 \pm(1.97)$ \\
\hline & Curvelet & $35.83( \pm 0.62)$ & $0.880( \pm 0.010)$ & $101.2 \pm(1.66)$ \\
\hline & DWT+DCT & $36.43( \pm 0.72)$ & $0.892( \pm 0.011)$ & $103.1 \pm(1.12)$ \\
\hline & DCT+Curvelet & $36.64( \pm 0.65)$ & $0.933( \pm 0.016)$ & $225.3 \pm(1.43)$ \\
\hline & DWT+Curvelet & $38.12( \pm 0.54)$ & $0.945( \pm 0.013)$ & $207.9 \pm(1.10)$ \\
\hline & DWT+DCT+Curvelet & $38.82( \pm 0.37)$ & $0.947( \pm 0.012)$ & $365.1 \pm(1.25)$ \\
\hline \multirow{7}{*}{ Peppers } & DCT & $34.19( \pm 0.21)$ & $0.849( \pm 0.019)$ & $53.20 \pm(1.11)$ \\
\hline & DWT & $35.60( \pm 0.47)$ & $0.866( \pm 0.014)$ & $41.88 \pm(1.29)$ \\
\hline & Curvelet & $36.28( \pm 0.54)$ & $0.871( \pm 0.013)$ & $100.3 \pm(1.53)$ \\
\hline & DWT+DCT & $37.97( \pm 0.82)$ & $0.885( \pm 0.011)$ & $102.9 \pm(0.97)$ \\
\hline & DCT+Curvelet & $38.22( \pm 0.75)$ & $0.886( \pm 0.018)$ & $227.8 \pm(1.99)$ \\
\hline & DWT+Curvelet & $39.86( \pm 0.44)$ & $0.950( \pm 0.012)$ & $205.6 \pm(0.94)$ \\
\hline & DWT+DCT+Curvelet & $40.22( \pm 0.38)$ & $0.952( \pm 0.011)$ & $365.2 \pm(0.87)$ \\
\hline \multirow{7}{*}{ Goldhill } & DCT & $28.33( \pm 0.39)$ & $0.849( \pm 0.014)$ & $54.13 \pm(1.98)$ \\
\hline & DWT & $29.26( \pm 0.55)$ & $0.855( \pm 0.008)$ & $42.25 \pm(1.22)$ \\
\hline & Curvelet & $31.22( \pm 0.73)$ & $0.864( \pm 0.007)$ & $101.2 \pm(0.93)$ \\
\hline & DWT+DCT & $32.74( \pm 0.18)$ & $0.875( \pm 0.008)$ & $104.4 \pm(1.01)$ \\
\hline & DCT+Curvelet & $32.93( \pm 0.25)$ & $0.876( \pm 0.010)$ & $228.2 \pm(0.93)$ \\
\hline & DWT+Curvelet & $34.38( \pm 0.24)$ & $0.889( \pm 0.007)$ & $210.6 \pm(1.67)$ \\
\hline & DWT+DCT+Curvelet & $35.29( \pm 0.20)$ & $0.890( \pm 0.005)$ & $366.7 \pm(1.98)$ \\
\hline
\end{tabular}

Table 2 gives the experimental results. From this table we can see that the performance of curvelet is better than DWT and discrete cosine transform (DCT). Besides, more sparsifying transforms involved in the CS model will lead to better reconstruction. However, incorporating more sparsifying transforms into matrix $W$ will result in a larger size of $W$ and lead to a higher recovery time. The PSNR and SSIM of DWT+DCT+Curvelet are only a little higher than that of DWT+Curvelet, but it takes much more time to reconstruct the image. 


\subsubsection{Statistical significance tests}

Inspired by the works in [29], two statistical significance tests are conducted to further verify the the impact of the sparseness model to the reconstruction performance in this subsection. The first one is the Friedman test which is a non-parametric test that makes no assumption about the distribution of the data. Similar to [29], this test is applied to $n=4$ datasets and $k=7$ sparseness models. The large value of $k(k>4)$ enables one to use the $\chi^{2}$ distribution to obtain the relevant $P$-values that are used to either reject or accept the null hypothesis[29]. The null hypothesis is that the mean PSNR of the DWT+DCT+Curvelet sparse model is equal to the other sparse models. Analogous null hypothesis is also used for the SSIM and the recovery time. The hypothesis is rejected when the $P$-value is smaller than the significance level (usually set as 0.05). Using the Matlab command friedman(X), we obtain $2.386 \times 10^{-4}$, $1.655 \times 10^{-2}, 6.8 \times 10^{-3}$ as the $P$-values for the PSNR, the SSIM and the recovery time, respectively. These $P$-values are smaller than the significance level of 0.05 , and so the null-hypothesis that the performance of the DWT+DCT+Curvelet sparse model is equal to the other sparse models is rejected.

The second statistical significance test is the post hoc Bonferroni-Dunn test which utilizes the critical values from the $t$ distribution. In this test, the significance level of 0.05 is commonly used. Similar to Friedman test, three null hypotheses are tested: the first is that the mean PSNR of the DWT+DCT+Curvelet sparse model is equal to all other sparse models, the second hypothesis is that the mean SSIM values of the DWT+DCT+Curvelet sparse model is equal to all other sparse models, the third hypothesis is that the mean recovery time of the DWT+DCT+Curvelet sparse model is equal to all other sparse models. Using the SPSS software, we obtain the results and 
show them in Table 3. It can be seen that the hypothesis that the mean PSNR and the SSIM values obtained by the DWT+DCT+Curvelet sparseness are equal to the DWT+Curvelet is not rejected while the the hypothesis that the mean recovery time of the DWT+DCT+Curvelet sparse model is equal to the DWT+Curvelet is rejected. These results are consistent in the experimental results in table 2. Based on the statistical significance test and in order to achieve a good tradeoff between the recovery quality and time, we use the combination of DWT and Curvelet as the sparsifying transform matrix.

Table 3 The results of Bonferroni-Dunn test, where $\times$ denotes rejection of the null hypothesis (no difference between DWT+DCT+Curvelet and the comparing sparseness model) and $\sqrt{ }$ denotes that the null hypothesis is not rejected.

\begin{tabular}{cccc}
\hline Sparse model & PSNR & SSIM & Recovery time \\
\hline DCT & $\times$ & $\times$ & $\times$ \\
DWT & $\times$ & $\times$ & $\times$ \\
Curvelet & $\times$ & $\sqrt{ }$ & $\times$ \\
DWT+DCT & $\times$ & $\times$ & $\times$ \\
DCT+Curvelet & $\times$ & $\sqrt{ }$ & $\times$ \\
DWT+Curvelet & $\sqrt{ }$ & $\sqrt{ }$ & $\times$ \\
\hline
\end{tabular}

\subsubsection{Compared with TV model}

In this experiment, the quality of the images reconstructed by the proposed algorithm using the FrTV combined with sparsifying transforms is tested, compared with the reconstruction algorithm using TV combined with sparsifying transforms. Reconstructed images of Barbara and Lena and their local enlargements are shown in Fig.9 and Fig.10. From the two figures, we can see that only using sparsifying transforms, the details of the recovered images are preserved, but it is not smooth. Using TV regularization combined with sparsifying transforms makes the recovered image quality sharper, but it loses some details and textures. While using FrTV combined with sparsifying transforms can efficiently reconstruct the fine details and improve the 


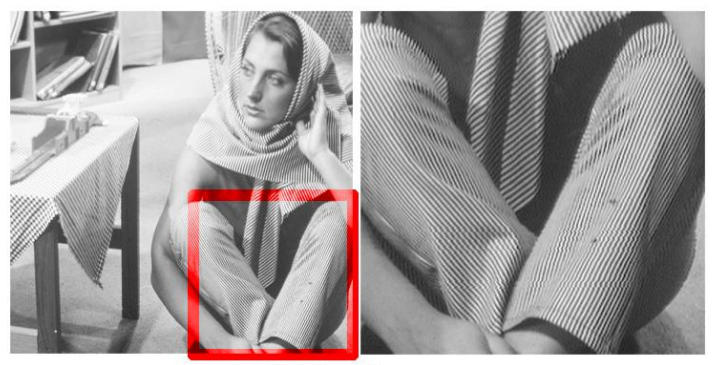

Original Image

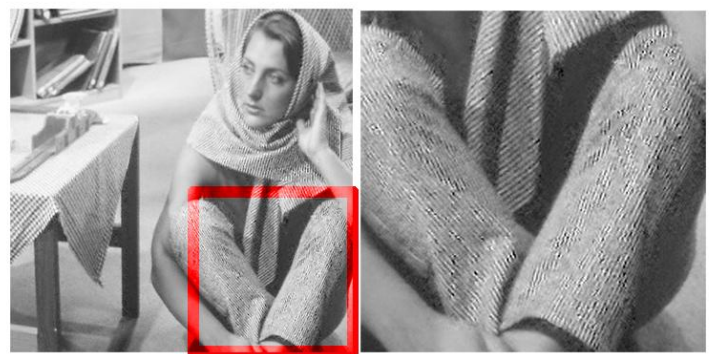

Curvelet $+\mathrm{TV}(\mathrm{PSNR}=28.37 \mathrm{~dB}, \mathrm{SSIM}=0.841)$

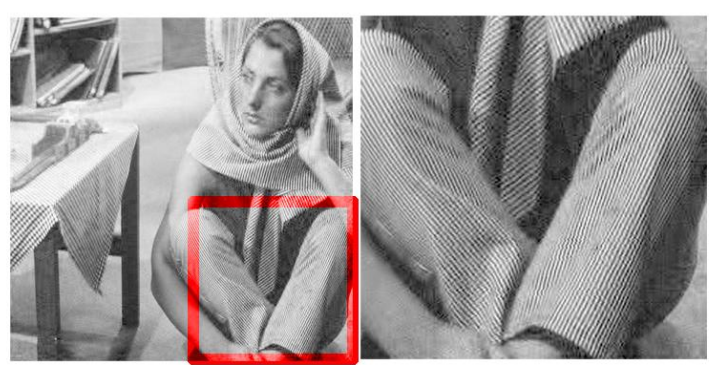

$\mathrm{DWT}+$ Curvelet $(\mathrm{PSNR}=28.79 \mathrm{~B}, \mathrm{SSIM}=0.847)$

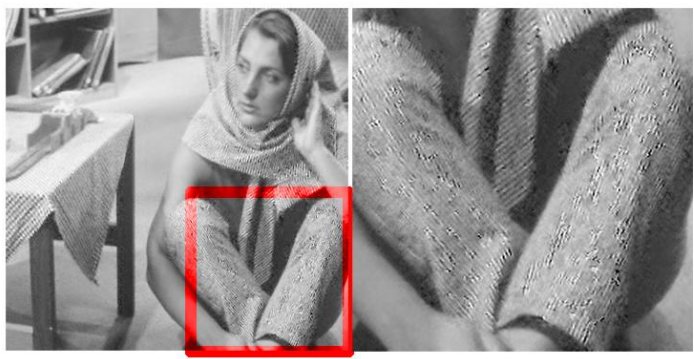

$\mathrm{DWT}+\mathrm{TV}(\mathrm{PSNR}=28.19 \mathrm{~dB}, \mathrm{SSIM}=0.803)$

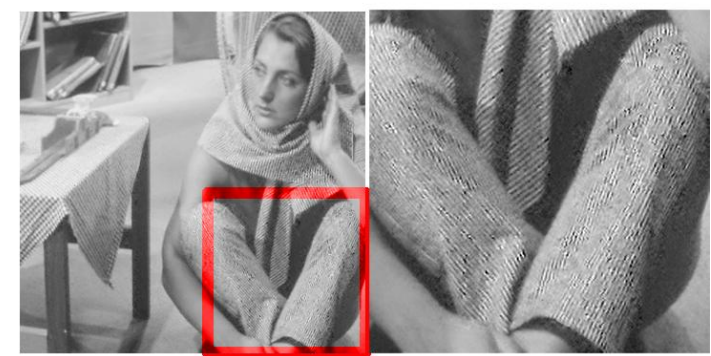

$\mathrm{DWT}+$ Curvelet $+\mathrm{TV}(\mathrm{PSNR}=28.96 \mathrm{~dB}, \mathrm{SSIM}=0.850)$

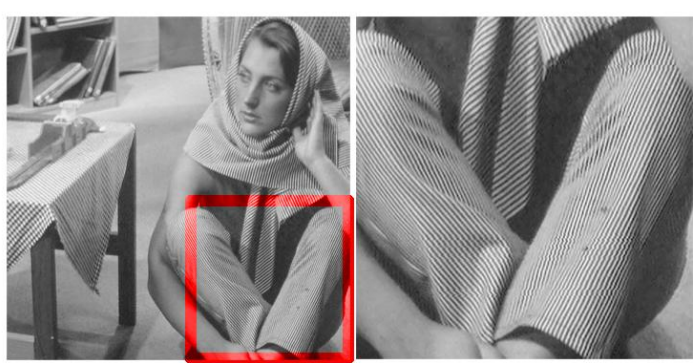

$\mathrm{DWT}+$ Curvelet $+\mathrm{FrTV}(\mathrm{PSNR}=34.74 \mathrm{~dB}, \mathrm{SSIM}=0.935)$

Fig.9 Reconstructed images (Barbara) with different sparseness models

quality of reconstructed image. The point and curve features are simultaneously reconstructed well, so it has the highest PNSR and SSIM.

\subsection{Comparison to other reconstruction algorithm.}

In this experiment, the quality of the images reconstructed by FrTVGP is tested, compared with several state-of-the-art TV based CS sparse image reconstruction algorithms: $\ell_{1}$-magic [7], NESTA[8], TVAL3[9], 2DPG[10]. For these algorithms, we choose the empirical parameter settings which give the best performance in the recovery quality in our experiment. In the experiment, various sampling ratios are used to validate the effectiveness of the proposed algorithm. In order to reduce the 

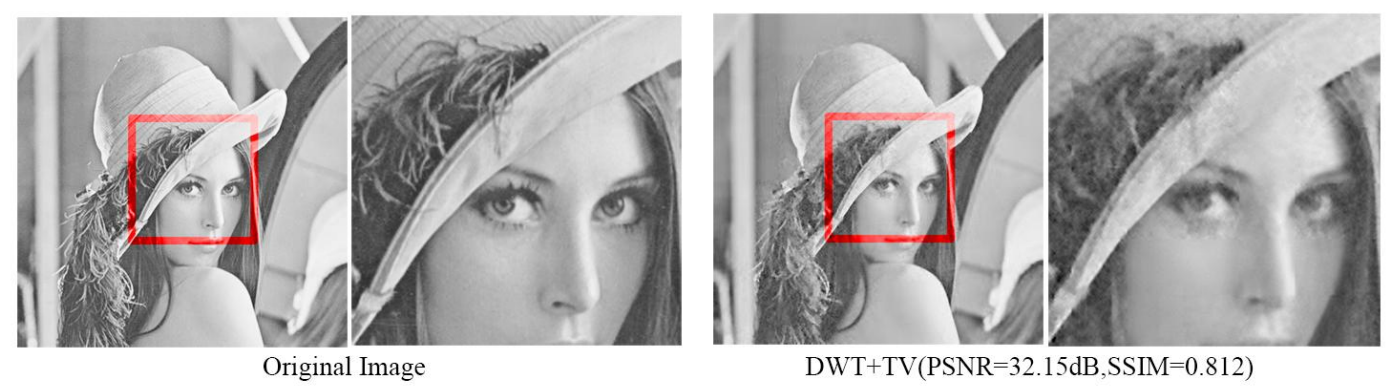

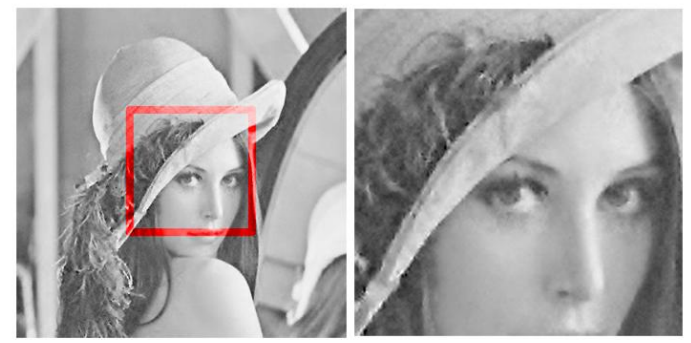

Curvelet $+\mathrm{TV}(\mathrm{PSNR}=34.03 \mathrm{~dB}, \mathrm{SSIM}=0.881)$

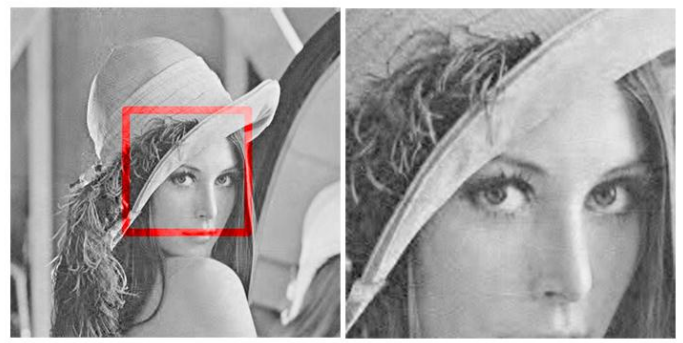

$\mathrm{DWT}+$ Curvelet(PSNR=35.38dB, SSIM=0.916)

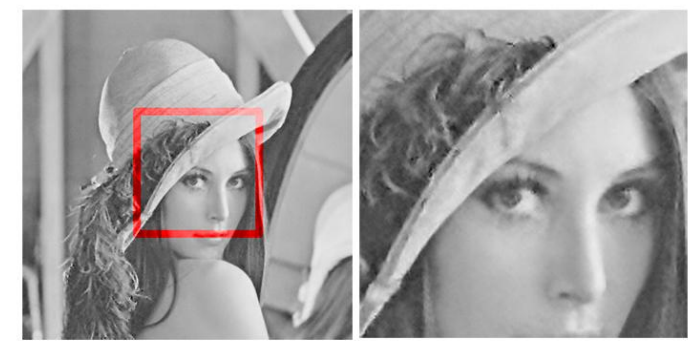

$\mathrm{DWT}+$ Curvelet $+\mathrm{TV}(\mathrm{PSNR}=35.14 \mathrm{~dB}, \mathrm{SSIM}=0.903)$

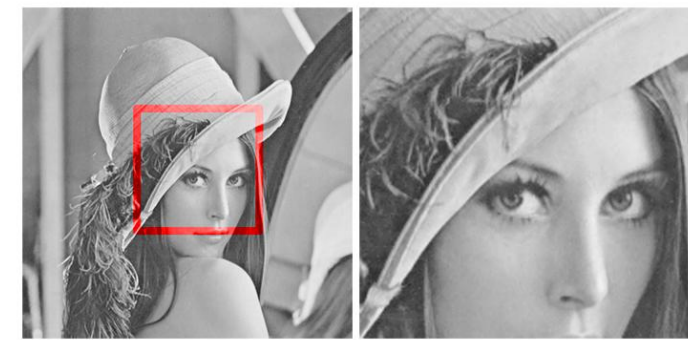

DWT + Curvelet + FrTV $($ PSNR $=37.97 \mathrm{~dB}, \mathrm{SSIM}=0.941)$

Fig.10 Reconstructed images (Lena) with different sparseness models
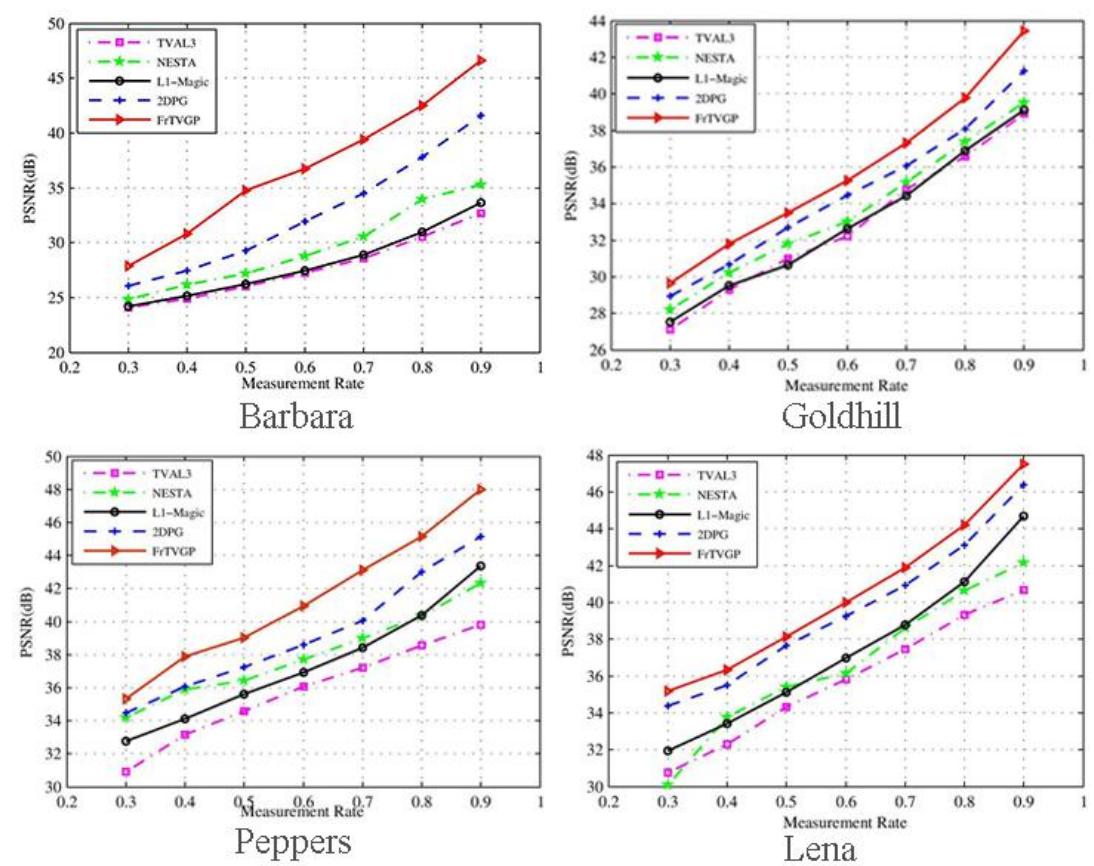

Fig.11 Comparison of PSNR values at different measurement rates.

computational complexity and memory requirements, images are divided into non- 

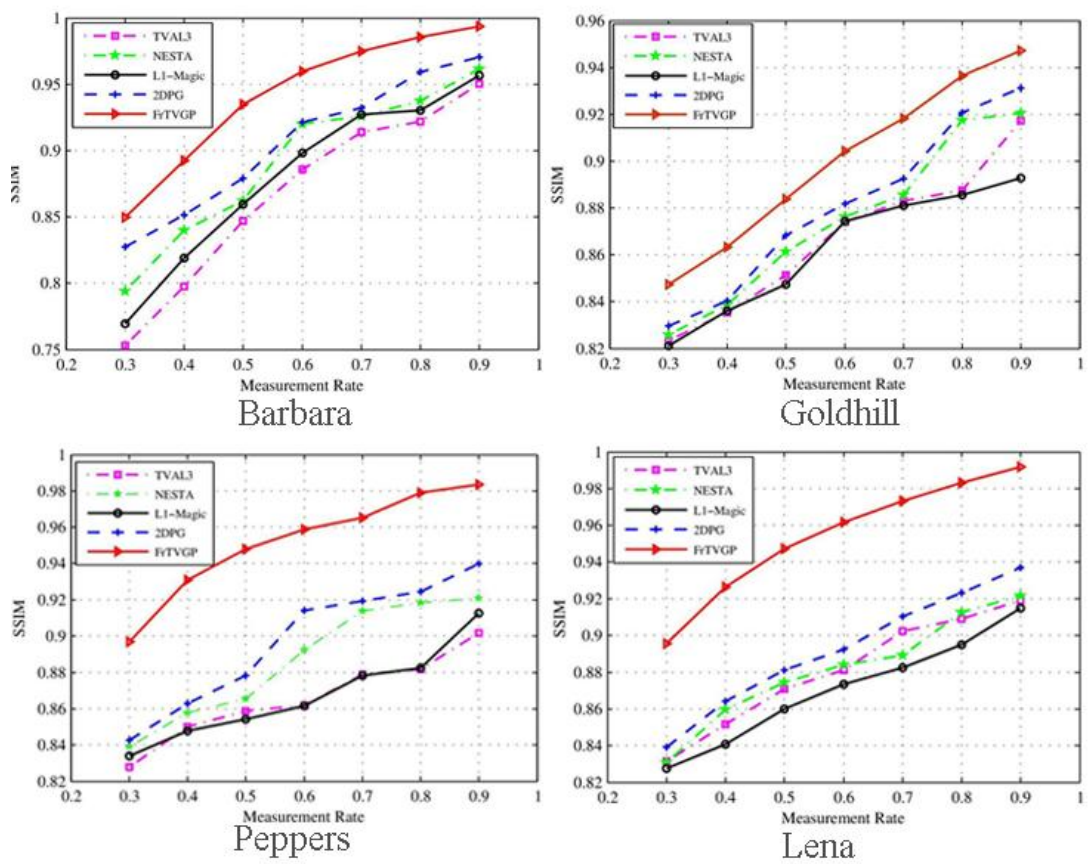

Fig.12 Comparison of SSIM values at different measurement rates.

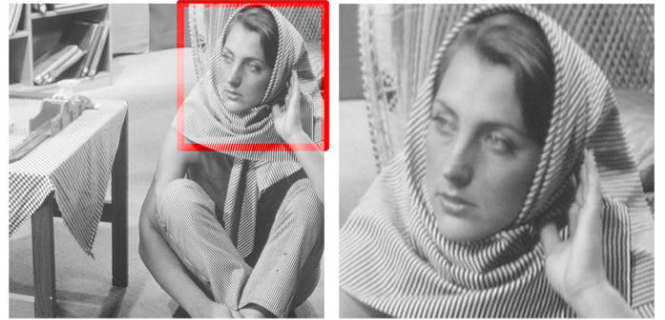

Original image

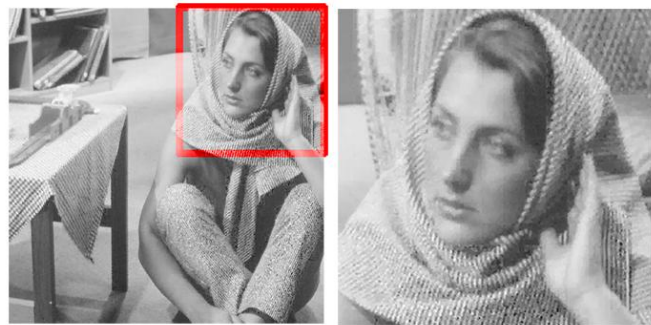

TVAL3(PSNR:26.03dB, SSIM:0.847)

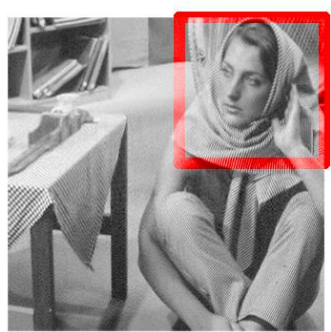

2DPG(PSNR:29.25dB, SSIM:0.879)

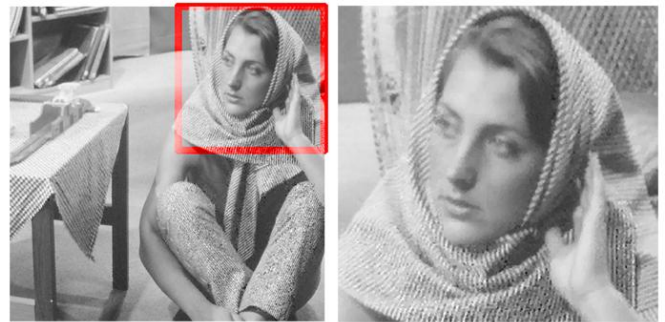

L1-magic(PSNR:26.24dB, SSIM:0.860)

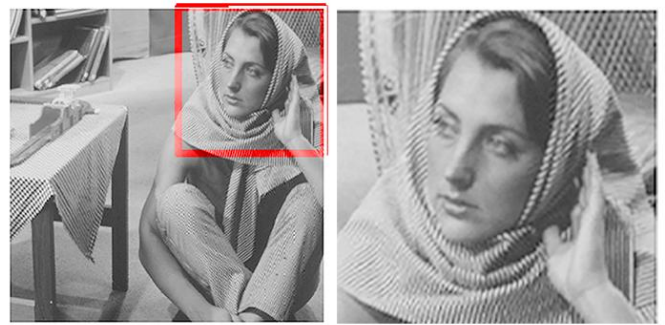

NESTA(PSNR:27.19dB, SSIM:0.862)

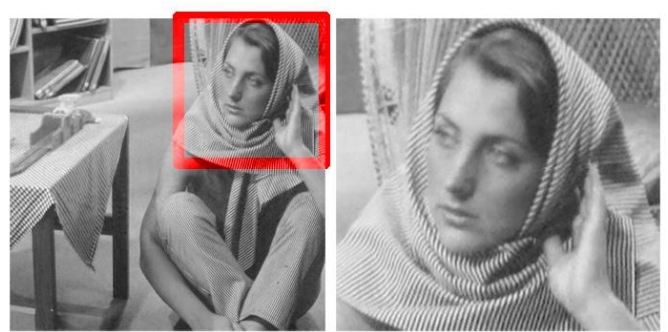

FrTVGP(PSNR:34.74dB, SSIM:0.935)

Fig.13 Reconstructed images (Barbara) with different reconstruction algorithm

overlapping blocks of size $16 \times 16$ for $\ell_{1}-$ magic, NESTA, TVAL3 while entire images 


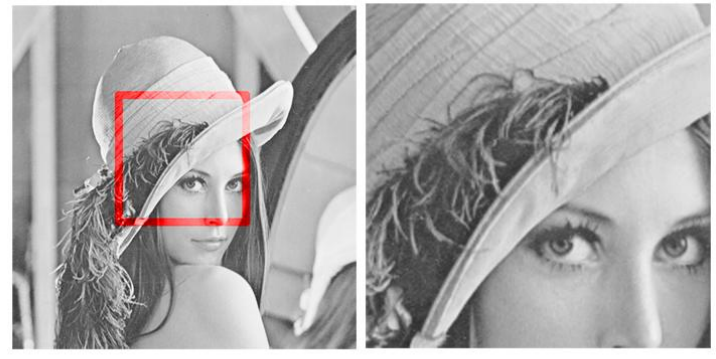

Original image

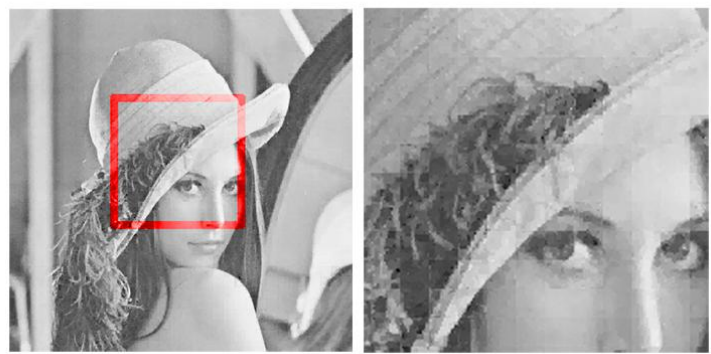

TVAL3(PSNR:34.32dB, SSIM:0.871)

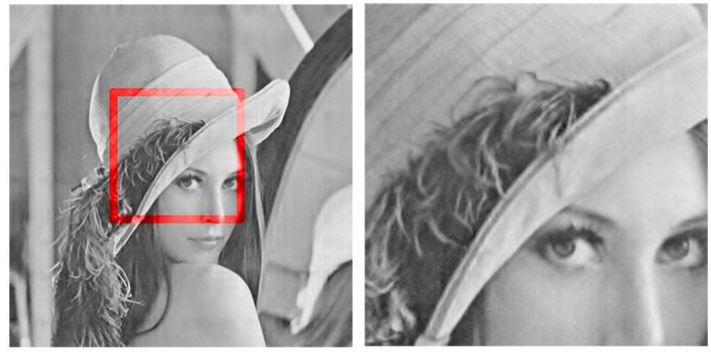

2DPG(PSNR:37.66dB, SSIM:0.881)

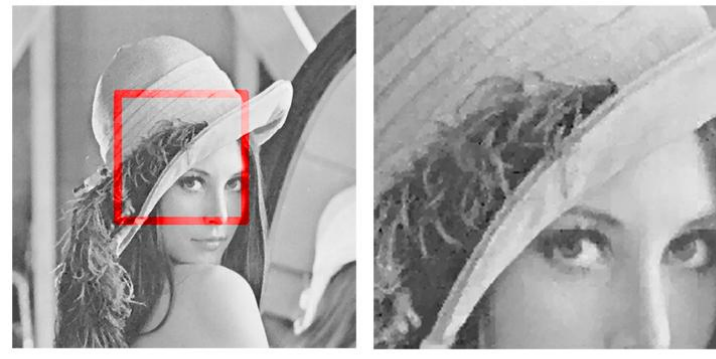

L1-magic(PSNR:35.11dB, SSIM:0.860)

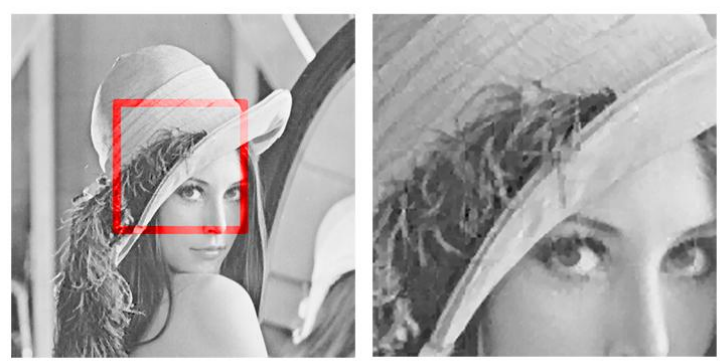

NESTA(PSNR:35.40dB, SSIM:0.875)

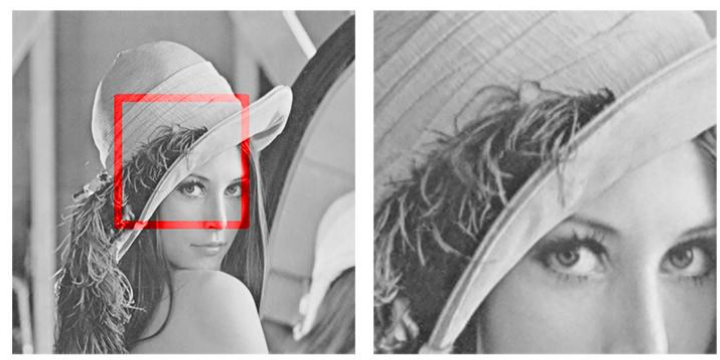

FrTVGP(PSNR:38.12dB, SSIM:0.947)

Fig.14 Reconstructed images (Lena) with different reconstruction algorithm

are considered for 2DPG and our proposed algorithm. In each sampling ratio, we also have repeated the experiment ten times and then calculated the average PSNR and SSIM. Fig. 11 displays their average PSNR values with the growth of the measurement rate for each image. Fig. 12 displays their average SSIM values with the growth of the measurement rate for each image. It can be seen that FrTVGP performs best for all of the images at different measurement rates. The subjective visual quality of the reconstructed images is used to further verify the effectiveness of the proposed algorithm. To compare the results visually, Fig. 13 -Fig.16 display some reconstructed images and local enlargements obtained using the five evaluated methods at $50 \%$ 


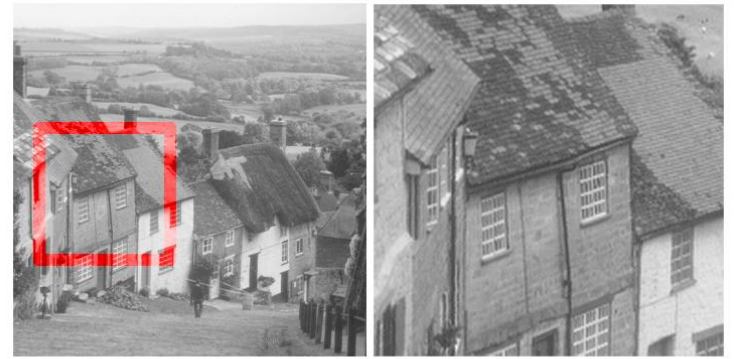

Original image

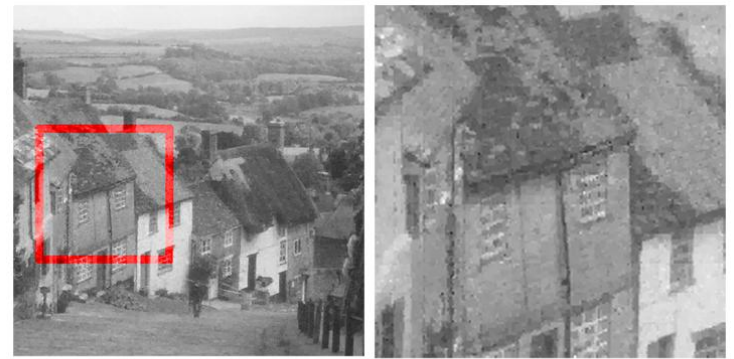

TVAL3(PSNR:30.99dB, SSIM:0.851
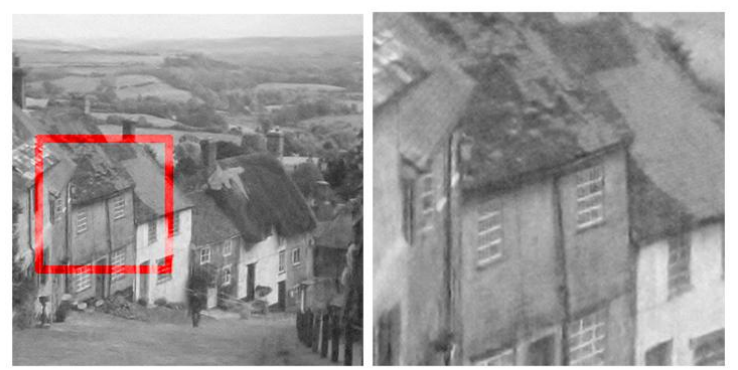

2DPG(PSNR:32.70dB, SSIM:0.868)

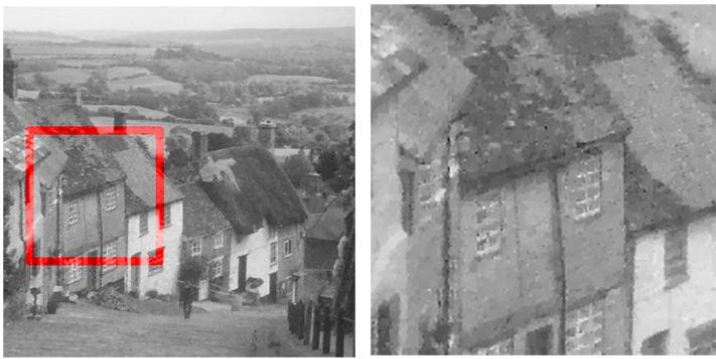

L1-magic(PSNR:30.64dB, SSIM:0.847)

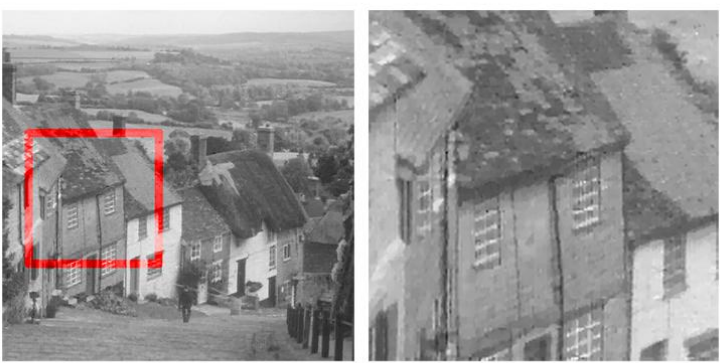

NESTA(PSNR:31.80dB, SSIM:0.861)
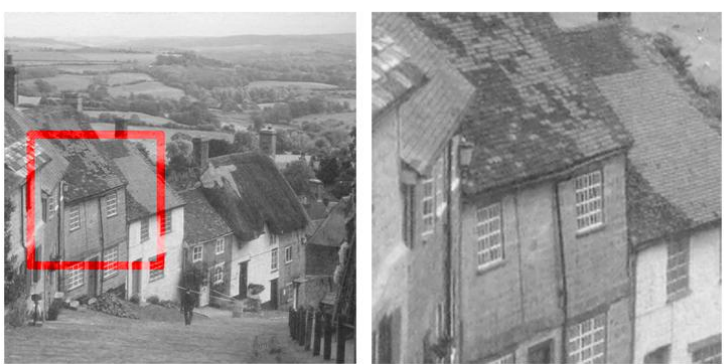

FrTVGP(PSNR:33.48dB, SSIM:0.884)

Fig.15 Reconstructed images (Goldhill) with different reconstruction algorithm

measurement rate. We can clearly see that the visual quality of the images recovered by FrTVGP is better than that of others, FrTVGP could efficiently reconstruct fine details and textures while preserving sharp edges.

\subsection{Computational complexity experiment}

In this experiment, the computational complexity of the proposed algorithm is tested, compared with the other four state-of-the-art TV based reconstruction algorithms. CPU time is used as a rough estimation of computational complexity. The experiment is performed in MATLAB 7.10 environment using an Intel Core 2 Duo, 2.1 $\mathrm{GHz}$ processor with $1 \mathrm{~GB}$ of memory, and under Microsoft Windows XP operating 


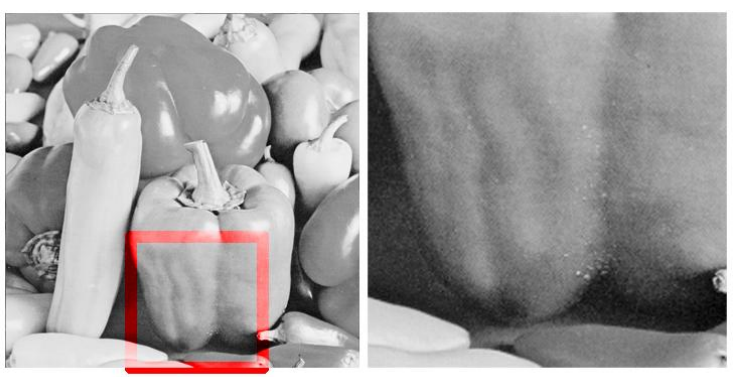

Original image

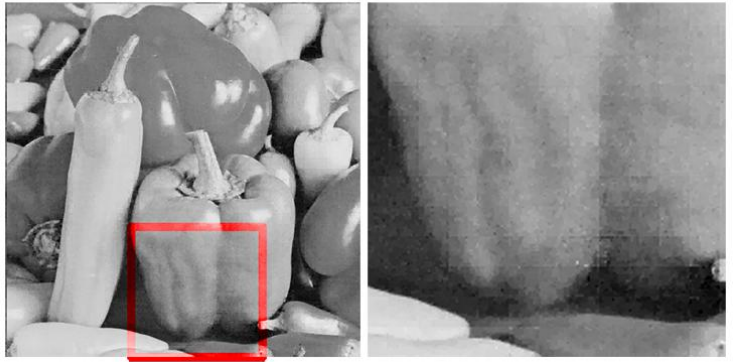

TVAL3(PSNR:34.57dB, SSIM:0.859)

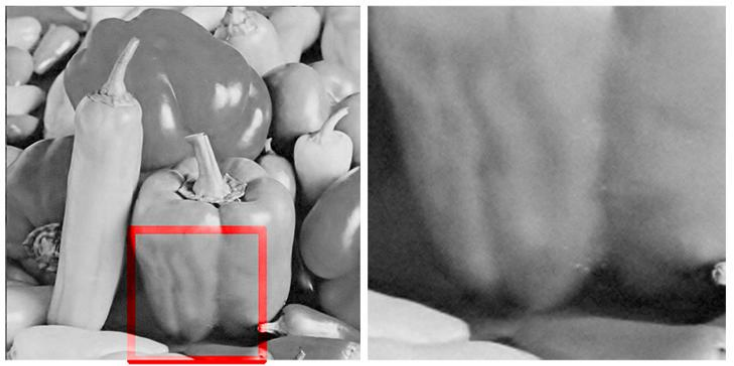

2DPG(PSNR:37.25dB, SSIM:0.878)
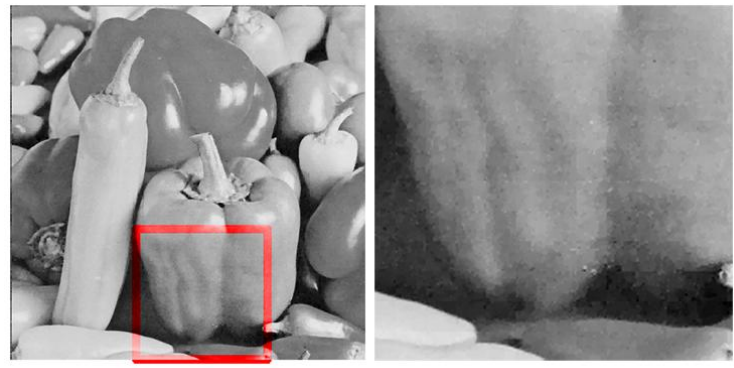

L1-magic(PSNR:35.59dB, SSIM:0.854)
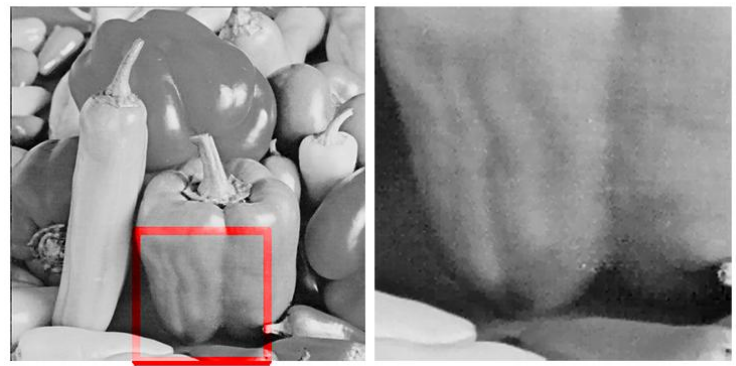

NESTA(PSNR:36.41dB, SSIM:0.865)
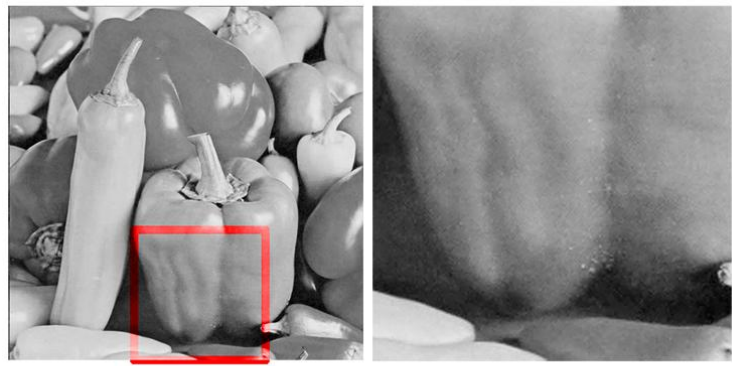

FrTVGP(PSNR:39.03dB, SSIM:0.948)

Fig.16 Reconstructed images (Peppers) with different reconstruction algorithm

Table 4 Average CPU time (s) of various reconstruction algorithms for all the test images at $50 \%$ measurement rate

\begin{tabular}{cccccc}
\hline Image & L1-magic & TVAL3 & NESTA & 2DPG & FrTVGP \\
\hline Barbara & 914.6 & 90.4 & 220.5 & 70.7 & $\mathbf{2 0 7 . 4}$ \\
Lena & 915.2 & 91.6 & 223.7 & 71.6 & $\mathbf{2 0 9 . 7}$ \\
Goldhill & 918.3 & 93.7 & 221.6 & 73.2 & $\mathbf{2 1 1 . 3}$ \\
Peppers & 909.8 & 95,2 & 225.7 & 72.5 & $\mathbf{2 0 8 . 6}$ \\
\hline
\end{tabular}

system. Table 4 gives the CPU time (s) required to recover four images by the five reconstruction algorithms at $50 \%$ measurement rates. From this table we can see that L1-magic algorithm has the highest computational complexity. Compared with TVAL3 algorithm and 2DPG algorithm, our algorithm has higher complexity. That is because 
additional computational overhead is added to FrTVGP with the inclusion of the updating of the regularization parameter. On average, the time computational complexity of FrTVGP is rough 3 times that of 2DPG. However, FrTVGP algrotithm has higher reconstruction quality.

\subsection{Reconstruction performance under different noise intensity}

In this experiment, we test the impact of the Gaussian noise or the speckle noise arising from the sensor to the reconstruction performance.

The images contaminated by the Gaussian noise can be formulated as:

$$
\mathbf{f}=\mathbf{f}_{\mathbf{0}}+\mathbf{n}_{\mathrm{g}}
$$

where $\mathbf{f}_{\mathbf{0}}$ is the original image, $\mathbf{f}$ is the noisy image, $\mathbf{n}_{\mathbf{g}}$ is the Gaussian noise and characterized by $\mathbf{n}_{\mathrm{g}} \sim N\left(0, \sigma^{2} I\right)$, where $\sigma$ is the standard deviation of Gaussian noise. Fig. 17 shows some reconstructed images of Peppers under different Gaussian noise intensity with $50 \%$ measurement rate. From this figure we can see that when $\sigma<20$, FrTVGP could still efficiently reconstruct the original images. However, when $\sigma>20$ the quality of the reconstructed images declines quickly, there are still some noise in the reconstructed images.

The images contaminated by the speckle noise can be formulated as:

$$
\mathbf{f}=\mathbf{f}_{\mathbf{0}}+\mathbf{n}_{p} \bullet \mathbf{f}_{\mathbf{0}}
$$

where $\mathbf{n}_{p}$ is uniformly distributed random noise with mean 0 and variance $v$. Fig. 18 shows some reconstructed images of Peppers under different speckle noise variance $v$ with $50 \%$ measurement rate. From this figure we can see that when $v<0.3$, FrTVGP could still efficiently reconstruct the original images. However, when $v>0.3$ the quality of the reconstructed images declines quickly, there are still some speckle noise 
in the reconstructed images.

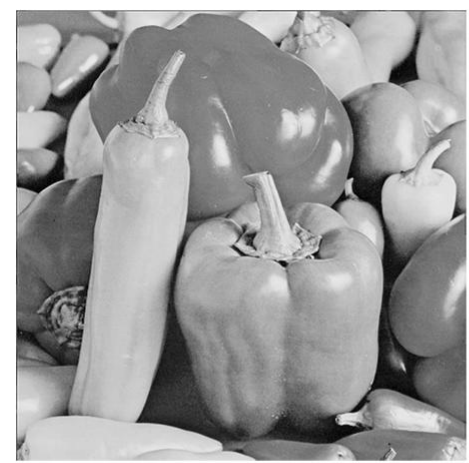

Original image

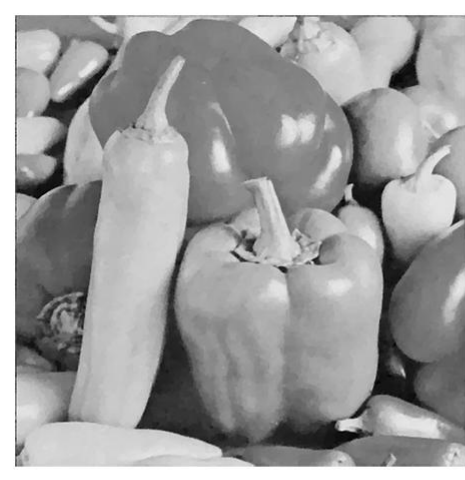

$\sigma=15$

$(\mathrm{PSNR}=31.71 \mathrm{~dB}, \mathrm{SSIM}=0.884)$

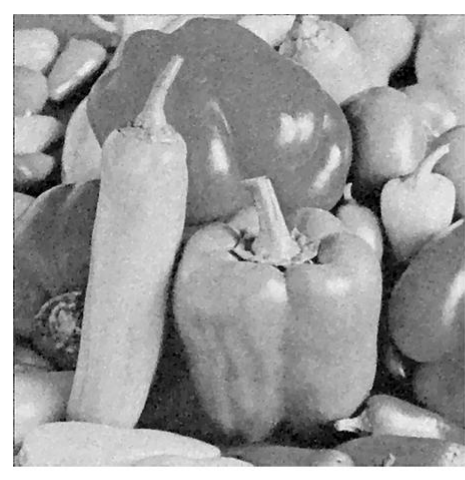

$\sigma=25$

$(\mathrm{PSNR}=28.78 \mathrm{~dB}, \mathrm{SSIM}=0.715)$

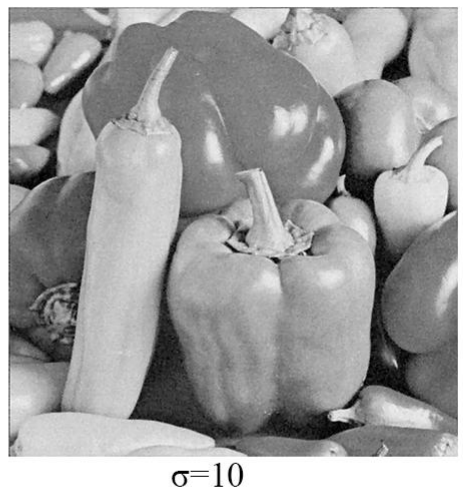

$(\mathrm{PSNR}=34.23 \mathrm{~dB}, \mathrm{SSIM}=0.894)$

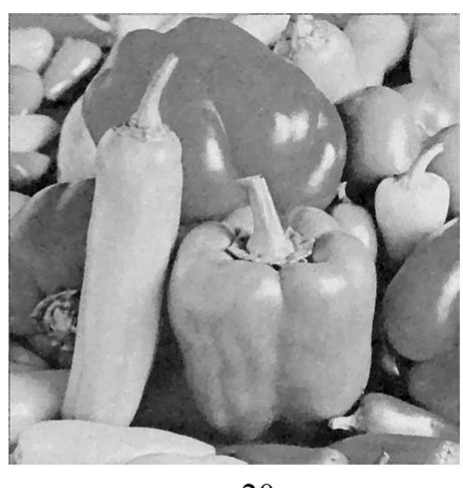

$\sigma=20$

$(\mathrm{PSNR}=30.46 \mathrm{~dB}, \mathrm{SSIM}=0.797)$

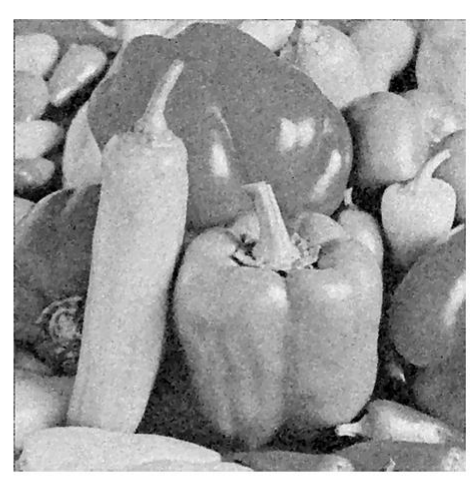

$$
\sigma=30
$$

$(\mathrm{PSNR}=26.85 \mathrm{~dB}, \mathrm{SSIM}=0.607)$

Fig.17 Reconstructed images (Peppers) with different Gaussian noise intensity

\section{Conclusions}




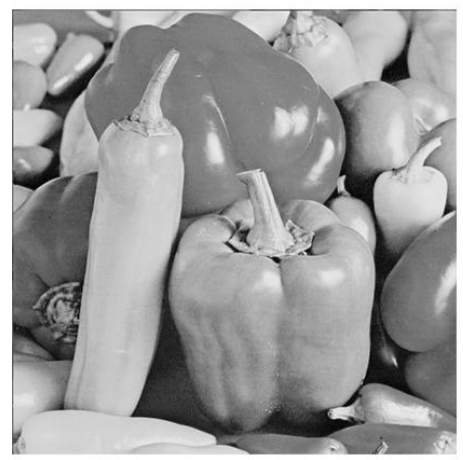

Original image

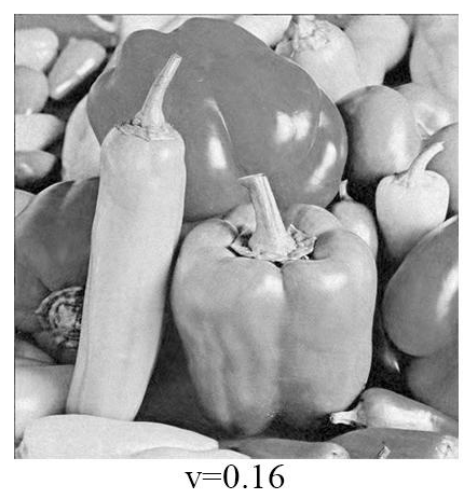

$(\mathrm{PSNR}=33.25 \mathrm{~dB}, \mathrm{SSIM}=0.912)$

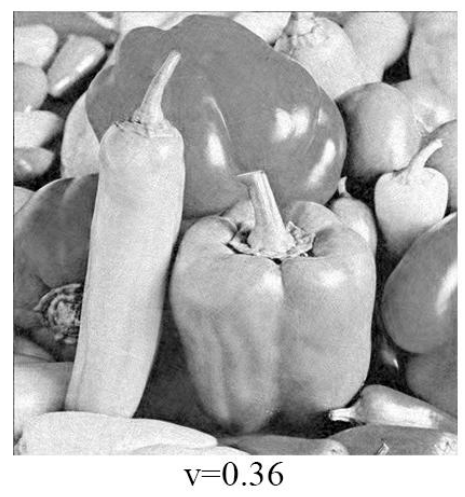

$(\mathrm{PSNR}=27.55 \mathrm{~dB}, \mathrm{SSIM}=0.801)$

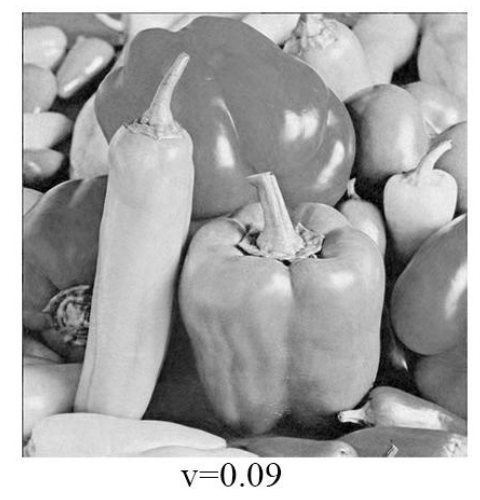

$(\mathrm{PSNR}=38.45 \mathrm{~dB}, \mathrm{SSIM}=0.937)$

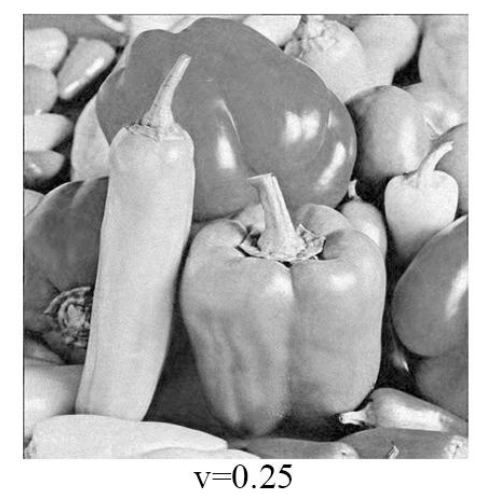

$(\mathrm{PSNR}=30.48 \mathrm{~dB}, \mathrm{SSIM}=0.887)$

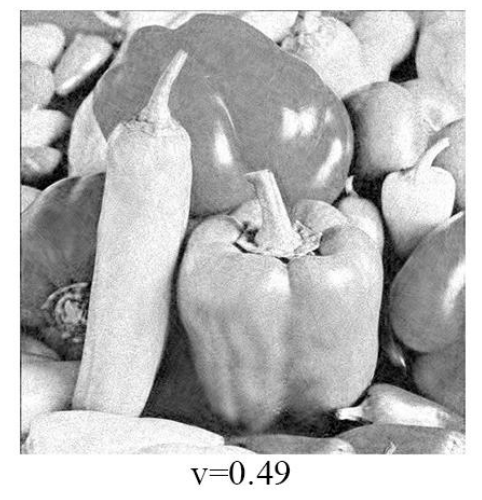

$(\mathrm{PSNR}=24.14 \mathrm{~dB}, \mathrm{SSIM}=0.724)$

Fig.18 Reconstructed images (Peppers) with different speckle noise variance

A 2DCS sparse image reconstruction model based on FrTV combined with DWT and curvelet transform is proposed in this paper. Furthermore, FrTVGP algorithm is developed to solve this combined FrTV and sparsifying transforms constrained 
minimization problem effectively. By taking the advantage of FrTV model and sparsifying transforms, image edges and fine details can be well reconstructed by the proposed algorithm. Experimental results show that, compared with several state-of-theart CS based image reconstruction algorithms, the reconstructed images obtained by the proposed approach not only have higher peak-signal-to-noise (PSNR) values, but also have better visual effects.

\section{Acknowledgements}

This work was supported by National Science Foundation of P.R.China (Grant: 61271341) and the Sichuan Science Technology Foundation (Grant:2013JY0136).

\section{Reference}

[1] D. L. Donoho, Compressed sensing, IEEE Trans. Inf. Theory, 52(4)(2006)12891306.

[2] R. G. Baraniuk, Compressive sensing, IEEE Signal Process. Mag., 24(4)(2007) 118122.

[3]C. Deng, W. Lin, B.-S. Lee, et al, Robust image coding based upon compressive sensing, IEEE Trans. Multimedia 14 (2) (2012) 278-290.

[4]A.H Wang, B. Zeng, H. Chen, Wireless multicasting of video signals based on distributed compressed sensing, Signal Processing: Image Communication 29(5)(2014) 599-606.

[5] M. F. Duarte, R.G. Baraniuk. Kronecker compressive sensing, IEEE Trans. Image Process., 21(2) (2012) 494-504.

[6] L. Rudin, S. Osher, E. Fatemi, Nonlinear total variation based noise removal algorithms, Physica D: Nonlinear Phenomena 60 (1992) 259-268.

[7] L1-Magic Toolbox ,available at http://users.ece.gatech.edu/ justin/l1magic/. 
[8]S. Becker, J. Bobin, E. Candès, NESTA: a fast and accurate first-order method for sparse recovery, SIAM J. Imaging Sci., 4(1)(2011) 1-39.

[9] C. Li, W. Yin, Y. Zhang, TVAL3: TV Minimization by augmented lagrangian and alternating direction algorithm, available at http://www.caam.rice.edu/ optimization/L1/TVAL3, (Online) 2009

[10 ] G. Chen, D.F Li, J.S,Zhang, Iterative gradient projection algorithm for twodimensional compressive sensing sparse image reconstruction, Signal Process., 104(2014)15-26

[11] I.Daubechies, The wavelet transform, time-frequency localization and signal analysis, IEEE Trans. Information Theory, 36(5)(1990) 961-1005.

[12] X.B Qu, X.Cao, D.Guo, et al, Combined sparsifying transforms for compressed sensing MRI, Electronics Letters , 46(2)(2010)121-123.

[ 13 ] E.Candès, D.Donoho, Curvelets- A surprisingly effective nonadaptive representation for objects with edges, Curves and Surface Fitting: Saint-Malo, Cohen, A., Rabut, C., andSchumaker, L. (Eds.), Vanderbilt Univ. Press, Nashville, 2000,pp.105-120.

[ 14 ] M.N. Do, M.Vetterli, The contourlet transform: An efficient directional multiresolution image representation, IEEE Trans. Image Process., 14(12)(2005), 2091-2106

[ 15 ] X.B Qu, X.Cao, D.Guo, et al, Compressed sensing MRI with combined sparsifying transforms and smoothed 10 norm minimization, in: Proc. Int. Conf. On Acoustics Speech and Signal Process, pp. 626-629,2010.

[16] J. Bai, X.C.Feng, Fractional-order anisotropic diffusion for image denoising, IEEE Trans. Image Process.16(10)(2007) 2492-2502.

[17] J. Zhang, Z.H.Wei, A class of fractional-order multi-scale variational models and alternating projection algorithm for image denoising, Appl. Math.Model.35(5)(2011)2516-2528. 
[18] D. Tian, D.Y. Xue, D.H. Wang, A fractional-order adaptive regularization primaldual algorithm for image denoising, Information Sciences 296 (2015) 147-159.

[19] S.B. Chen, D. L.Donoho, M.A. Saunders, Atomic decomposition by basis pursuit, SIAM Journal on Scientific Computing, 20(1)(1998) 33-61.

[20] S. Mallat, Z. Zhang, Matching pursuit with time-frequency dictionaries, IEEE Trans. Signal Process, 41(2)(1993) 3397-3415

[21] J Tropp, A Gilbert, Signal recovery from random measurements via orthogonal matching pursuit, IEEE Trans. Inform.Theory, 53(12)(2007) 4655-4666.

[ 22 ] A.Eftekhari, M.Babaie-Zadeh, H.A.Moghaddam, Two-dimensional random projection, Signal Process., 91(7)(2011) 1589-1603

[23 ] L. Liao, Y.N. Zhang and C. Zhang, 2DCS: Two dimensional random underdetermined projection for image representation and classification, in: Proceedings of the International Conference on Multimedia Technology(ICMT),July2011,pp.1-5.

[24]M. Lysaker, S. Osher, X.-C. Tai, Noise removal using smoothed normals and surface fitting, IEEE Trans. Image Process.13(10) (2004), 1345 - 1357.

[25]Z.M. Ren, C.J.He, Q.F.Zhang, Fractional order total variation regularization for image super-resolution, Signal Process., 93(2013)2408-2421.

[26] Spot Toolbox , available at http://www.cs.ubc.ca/labs/scl/spot

[27] G. Marjanovic, V. Solo, Sparse signal estimation with nonlinear conjugate gradients, in: Proc. Int. Conf. On Acoustics Speech and Signal Process, pp. 37663769,2010 .

[28] Z.Wang, A.C.Bovik, H.R. Sheikh, E.P. Simoncelli, Image quality assessment: from error visibility to structural similarity, IEEE Trans. Image Process., 13(4) (2004) 600-612

[29]A.P. James, S,Dimitrijev. Nearest neighbor classifier based on nearest feature decisions[J]. The Computer Journal, 2012: bxs001. 